\title{
CONIC SINGULARITIES OF SOLUTIONS TO PROBLEMS IN HYDRODYNAMICS OF A VISCOUS FLUID WITH A FREE SURFACE
}

\author{
V. A. KOZLOV, V. MAZ'YA and J. ROßMANN
}

\section{Introduction.}

The present paper is concerned with some boundary value problems for the Stokes system

$$
-\Delta U+\operatorname{grad} P=F, \quad \operatorname{div} U=0
$$

in a three-dimensional domain with conic points at the boundary. It is wellknown (see e.g. [5], [13]) that the solution $(U, P)$ of an elliptic boundary value problem for the system $(0.1)$ in a neighbourhood of a vertex $x^{(0)}=0$ of a cone $\mathscr{K}$ asymptotically behaves like a linear combination of terms of the form

$$
r^{z} \sum_{k=0}^{s} \frac{1}{k !}(\log r)^{k}\left(\begin{array}{c}
u^{(s-k)}(\omega) \\
r^{-1} p^{(s-k)}(\omega)
\end{array}\right)
$$

where $r=|x|$ and $\omega$ are coordinates on the sphere $S^{2}=\left\{x \in \mathrm{R}^{3}:|x|=1\right\}$. In order to obtain this result, one applies the Mellin transform $\mathscr{M}_{r \rightarrow \lambda}, \lambda \in \mathrm{C}$, to the operator of the boundary value problem in $\mathscr{K}$. In this way a pencil $\mathfrak{A}$ of boundary value problems on the spherical domain $\mathscr{K} \cap S^{2}$ is generated. The above mentioned asymptotic representation follows by applying the Cauchy residual theorem to the inverse Mellin transform of the resolvent $\mathfrak{A}(\lambda)^{-1}$. The exponents $z$ in (0.2) form a discrete sequence of eigenvalues of $\mathfrak{H}(\lambda)$, while $\left(u^{(0)}, p^{(0)}\right)$ is an eigenvector and $\left(u^{(1)}, p^{(1)}\right), \ldots,\left(u^{(s)}, p^{(s)}\right)$ are generalized eigenvectors corresponding to $z$. Thus, the question of conic singularities has been reduced to the spectral analysis of the operator pencil $\mathfrak{A}(\lambda)$ which is, in fact, the subject of the present paper.

We consider the following boundary conditions for the system $(0.1)$ :

Received April 1, 1996. 
(i) $U=0$,

(ii) $U_{n}=0, \quad \varepsilon_{n, \tau}(U)=0$,

(iii) $U_{\tau}=0, \quad-P+2 \varepsilon_{n, n}(U)=0$,

where $n=\left(n_{1}, n_{2}, n_{3}\right)$ is the exterior normal to $\partial \mathscr{K}, U_{n}=U \cdot n$ is the normal component of the velocity $U=\left(U_{1}, U_{2}, U_{3}\right), U_{\tau}$ is the tangential component of the vector $U$ (i.e., the projection of $U$ onto the tangent plane to $\partial \mathscr{K}$ ), and $\varepsilon$ is the strain tensor. We admit that different boundary conditions may be prescribed on different conic subdomains of $\partial \mathscr{K}$. These boundary conditions appear in analysis of the steady-state motion of a viscous fluid in a vessel with a free surface having contact with the walls of the vessel (see for example [15]).

Here we list the main results of the paper:

1. The strip $\{\lambda:-1<\operatorname{Re} \lambda<0\}$ does not contain eigenvalues of the pencil $\mathfrak{A}$.

2. The strip $-2 \leq \operatorname{Re} \lambda \leq 1$ contains only real eigenvalues.

3. There are no generalized eigenvectors to the eigenvalues in the strip $-2<\operatorname{Re} \lambda<1$.

4. The numbers 0 and -1 are eigenvalues of $\mathfrak{A}$ if and only if there exists a nonzero constant vector $(u, p)=(c, 0)$ satisfying the boundary conditions.

5. We describe all eigenvectors and generalized eigenvectors corresponding to $\lambda=1$, when this number is an eigenvalue of $\mathfrak{U}(\lambda)$.

6. We derive a variational principle for the eigenvalues of $\mathfrak{A}$ in the interval $[0,1)$ and find the total multiplicity of the spectrum in $[0,1)$. In the case of the boundary conditions (i) and (iii) on different parts of $\partial \mathscr{K}$, this multiplicity is an increasing function of $\mathscr{K}$ (see Corollary 8.1).

In [11] similar results were obtained for the system (0.1) with the Dirichlet boundary conditions. We remark that the Neumann condition

$$
-P+2 \varepsilon_{n, n}(U)=0, \quad \varepsilon_{n, \tau}(U)=0 \quad \text { on } \partial \mathscr{K}
$$

which appears in the hydrodynamic potential theory is not considered in the present paper. For this problem it was shown in [8] that the strip $-1 \leq \operatorname{Re} \lambda \leq 0$ contains only the eigenvalues -1 and 0 , if $\partial \mathscr{K}$ admits a oneto-one orthogonal projection onto a plane.

We give some consequences of our results for solutions of the mixed boundary value problems under consideration in a bounded domain of polyhedral type. Using the same technique as in [5], one can show that the solution $(U, P)$ has the asymptotics 


$$
\begin{aligned}
& U=\sum_{k} c_{k} r^{\lambda_{k}} u^{(k)}(\omega)+O(r), \\
& P=\sum_{k} c_{k} r^{\lambda_{k}-1} p^{(k)}(\omega)+O(\log r)
\end{aligned}
$$

near every singular boundary point $x^{(0)}$, where $\lambda_{k}$ are eigenvalues of $\mathfrak{A}(\lambda)$ in the interval $[0,1)$. (Here we assume for simplicity that the right-hand sides in the Stokes system and the boundary conditions vanish near $x^{(0)}$.) In comparison with the general asymptotic representation (0.2), the asymptotics just written does not contain logarithmic terms and the powers of $r$ are necessarily real.

As applications of our results, we consider two special cases in Section 9.

\section{Formulation of the problem.}

Let $\mathscr{K}$ be the cone $\left\{x=\left(x_{1}, x_{2}, x_{3}\right) \in \mathrm{R}^{3}: x /|x| \in \Omega\right\}$, where $\Omega$ is a domain on the unit sphere with Lipschitz boundary $\partial \Omega=\bar{\gamma}_{1} \cup \ldots \cup \bar{\gamma}_{N}$. Here $\gamma_{1}, \ldots, \gamma_{N}$ are pairwise disjoint open connected arcs of $\partial \Omega$. Then the boundary of $\mathscr{K}$ is the union of faces, $\partial \mathscr{K}=\bar{\Gamma}_{1} \cup \cdots \cup \bar{\Gamma}_{N}$, where $\Gamma_{k}=\left\{x: x /|x| \in \gamma_{k}\right\}$.

We divide the set of the indices $1, \ldots, N$ into three subsets $I_{0}, I_{n}, I_{\tau}$. Our goal is to find solutions of the system

$$
-\Delta U+\operatorname{grad} P=0, \quad \operatorname{div} U=0 \quad \text { in } \mathscr{K}
$$

satisfying the boundary conditions

$$
\left\{\begin{array}{l}
U=0 \text { on } \Gamma_{k} \text { for } k \in I_{0}, \\
U_{n}=0, \varepsilon_{n, \tau}(U)=0 \text { on } \Gamma_{k} \text { for } k \in I_{n}, \\
U_{\tau}=0,-P+2 \sum_{i, j=1}^{3} \varepsilon_{i j}(U) n_{i} n_{j}=0 \text { on } \Gamma_{k} \text { for } k \in I_{\tau},
\end{array}\right.
$$

which will be understood in the generalized sense.

In order to introduce the notion of generalized solutions, we need the following Green formula 


$$
\begin{aligned}
& \int_{\mathscr{K}}\left(2 \sum_{i, j=1}^{3} \varepsilon_{i j}(U) \cdot \varepsilon_{i j}(\bar{V})-P \operatorname{div} \bar{V}-\operatorname{div} U \cdot \bar{Q}\right) d x \\
& =\int_{\mathscr{K}}((-\Delta U-\operatorname{grad} \operatorname{div} U+\operatorname{grad} P) \bar{V}-\operatorname{div} U \cdot \bar{Q}) d x \\
& +\sum_{j=1}^{3} \int_{\partial \mathscr{K} \backslash\{0\}}\left(-P n_{j}+2 \sum_{i=1}^{3} \varepsilon_{i j}(U) n_{i}\right) \bar{V}_{j} d \sigma,
\end{aligned}
$$

where

$$
\varepsilon_{i j}(U)=\frac{1}{2}\left(\partial_{x_{j}} U_{i}+\partial_{x_{i}} U_{j}\right) .
$$

If $(U, P)$ is a formal solution of the problem $(1.1),(1.2),(V, Q)$ vanishes for large and small $|x|$, and $V$ satisfies the conditions

$$
\begin{gathered}
V=0 \text { on } \Gamma_{k} \text { for } k \in I_{0}, \quad V_{n}=0 \text { on } \Gamma_{k} \text { for } k \in I_{n}, \\
V_{\tau}=0 \text { on } \Gamma_{k} \text { for } k \in I_{\tau},
\end{gathered}
$$

then (1.3) implies

$$
\int_{\mathscr{K}}\left(\sum_{i, j=1}^{3} \varepsilon_{i j}(U) \cdot \varepsilon_{i j}(\bar{V})-\frac{1}{2} P \operatorname{div} \bar{V}-\frac{1}{2} \operatorname{div} U \cdot \bar{Q}\right) d x=0
$$

Hence it is natural to define generalized solutions by means of this integral identity.

More precisely, let $\mathscr{H}$ be the space of all vector-functions $u \in H^{1}(\Omega)^{3}$ satisfying the conditions

(i) $u=0$ on $\gamma_{k}$ for $k \in I_{0}$,

(ii) $u_{n}=0$ on $\gamma_{k}$ for $k \in I_{n}$,

(iii) $u_{\tau}=0$ on $\gamma_{k}$ for $k \in I_{\tau}$,

where $u_{n}=u \cdot n, n$ denotes the exterior normal to $\partial \mathscr{K}$, and $u_{\tau}$ is the projection of the vector $u$ onto the tangent plane to $\mathscr{K}$. We say that the pair $(U, P)$ of the form

$$
\begin{aligned}
& U(x)=r^{\lambda_{0}} \sum_{k=0}^{s} \frac{1}{k !}(\log r)^{k} u^{(s-k)}(\omega), \quad u^{(s-k)} \in \mathscr{H}, \\
& P(x)=r^{\lambda_{0}-1} \sum_{k=0}^{s} \frac{1}{k !}(\log r)^{k} p^{(s-k)}(\omega), \quad p^{(s-k)} \in L_{2}(\Omega),
\end{aligned}
$$

is a generalized solution of (1.1), (1.2), if (1.5) is satisfied for all 
$(V, Q) \in H^{1}(\mathscr{K})^{3} \times L_{2}(\mathscr{K})$ with compact support in $\overline{\mathscr{K}} \backslash\{0\}$ such that $V$ satisfies the boundary conditions (1.4).

\section{The operator pencil generated by the boundary value problem.}

We rewrite the integral identity (1.5) in terms of the spherical components $U_{r}, U_{\varphi}, U_{\theta}$,

$$
\left(\begin{array}{l}
U_{r} \\
U_{\theta} \\
U_{\varphi}
\end{array}\right)=J\left(\begin{array}{l}
U_{1} \\
U_{2} \\
U_{3}
\end{array}\right), \quad J=J(\theta, \varphi)=\left(\begin{array}{ccc}
\sin \theta \cos \varphi & \sin \theta \sin \varphi & \cos \theta \\
\cos \theta \cos \varphi & \cos \theta \sin \varphi & -\sin \theta \\
-\sin \varphi & \cos \varphi & 0
\end{array}\right)
$$

of the vector $U$, where $r, \theta, \varphi$ are the spherical coordinates of the point $x=\left(x_{1}, x_{2}, x_{3}\right)$, i.e.,

$$
x_{1}=r \cos \varphi \sin \theta, \quad x_{2}=r \sin \varphi \sin \theta, \quad x_{3}=r \cos \theta .
$$

Then (1.5) takes the form

$$
\begin{aligned}
& \int_{0}^{\infty} \int_{\Omega}\left(\varepsilon_{r r}(U) \varepsilon_{r r}(\bar{V})+\varepsilon_{\theta \theta}(U) \varepsilon_{\theta \theta}(\bar{V})+\varepsilon_{\varphi \varphi}(U) \varepsilon_{\varphi \varphi}(\bar{V})+2 \varepsilon_{r \theta}(U) \varepsilon_{r \theta}(\bar{V})\right. \\
& +2 \varepsilon_{r \varphi}(U) \varepsilon_{r \varphi}(\bar{V})+2 \varepsilon_{\theta \varphi}(U) \varepsilon_{\theta \varphi}(\bar{V})-\frac{1}{2} P\left(\varepsilon_{r r}(\bar{V})+\varepsilon_{\theta \theta}(\bar{V})+\varepsilon_{\varphi \varphi}(\bar{V})\right) \\
& \left.-\frac{1}{2}\left(\varepsilon_{r r}(U)+\varepsilon_{\theta \theta}(U)+\varepsilon_{\varphi \varphi}(U)\right) \bar{Q}\right) r^{2} d \omega d r=0,
\end{aligned}
$$

where

$$
\left\{\begin{array}{l}
\varepsilon_{r r}=\partial_{r} U_{r}, \quad \varepsilon_{\varphi \varphi}=\frac{1}{r \sin \theta} \partial_{\varphi} U_{\varphi}+\frac{U_{r}}{r}+\cot \theta \frac{U_{\theta}}{r} \\
\varepsilon_{\theta \theta}=\frac{1}{r} \partial_{\theta} U_{\theta}+\frac{U_{r}}{r}, \quad \varepsilon_{r \varphi}=\frac{1}{2}\left(\frac{1}{r \sin \theta} \partial_{\varphi} U_{r}-\frac{U_{\varphi}}{r}+\partial_{r} U_{\varphi}\right), \\
\varepsilon_{r \theta}=\frac{1}{2}\left(\frac{1}{r} \partial_{\theta} U_{r}-\frac{U_{\theta}}{r}+\partial_{r} U_{\theta}\right), \\
\varepsilon_{\theta \varphi}=\frac{1}{2}\left(\frac{1}{r} \partial_{\theta} U_{\varphi}-\cot \theta \frac{U_{\varphi}}{r}+\frac{1}{r \sin \theta} \partial_{\varphi} U_{\theta}\right) .
\end{array}\right.
$$

We introduce the sesquilinear form 


$$
\begin{aligned}
& a\left(\left(\begin{array}{l}
u \\
p
\end{array}\right),\left(\begin{array}{l}
v \\
q
\end{array}\right) ; \lambda\right) \\
& =\frac{1}{\log 2} \int_{1}^{2} \int_{\Omega}\left(\varepsilon_{r r}(U) \varepsilon_{r r}(\bar{V})+\varepsilon_{\theta \theta}(U) \varepsilon_{\theta \theta}(\bar{V})+\varepsilon_{\varphi \varphi}(U) \varepsilon_{\varphi \varphi}(\bar{V})\right. \\
& +2 \varepsilon_{r \theta}(U) \varepsilon_{r \theta}(\bar{V})+2 \varepsilon_{r \varphi}(U) \varepsilon_{r \varphi}(\bar{V})+2 \varepsilon_{\theta \varphi}(U) \varepsilon_{\theta \varphi}(\bar{V}) \\
& -\frac{1}{2} P\left(\varepsilon_{r r}(\bar{V})+\varepsilon_{\theta \theta}(\bar{V})+\varepsilon_{\varphi \varphi}(\bar{V})\right) \\
& \left.-\frac{1}{2}\left(\varepsilon_{r r}(U)+\varepsilon_{\theta \theta}(U)+\varepsilon_{\varphi \varphi}(U)\right) \bar{Q}\right) r^{2} d \omega d r,
\end{aligned}
$$

where

$$
\begin{gathered}
U=r^{\lambda} u(\omega), \quad P=r^{\lambda-1} p(\omega), \quad V=r^{-1-\bar{\lambda}} v(\omega), \\
Q=r^{-2-\bar{\lambda}} q(\omega), d \omega=\sin \theta d \theta d \varphi
\end{gathered}
$$

Using (2.1), we find

$$
\begin{aligned}
& a\left(\left(\begin{array}{l}
u \\
p
\end{array}\right),\left(\begin{array}{l}
v \\
q
\end{array}\right) ; \lambda\right)=\left[u_{\omega}, v_{\omega}\right]+\int_{\Omega}\left(\frac{1}{2} \nabla_{\omega} u_{r} \cdot \nabla_{\omega} \bar{v}_{r}+(\lambda+2)(1-\lambda) u_{r} \bar{v}_{r}\right. \\
& +\frac{1}{2}(\lambda+2)(1-\lambda) u_{\omega} \cdot \bar{v}_{\omega}+u_{r} \nabla_{\omega} \cdot \bar{v}_{\omega}+\left(\nabla_{\omega} \cdot u_{\omega}\right) \bar{v}_{r}-\frac{1-\lambda}{2} u_{\omega} \cdot \nabla_{\omega} \bar{v}_{r} \\
& -\frac{\lambda+2}{2}\left(\nabla_{\omega} u_{r}\right) \cdot \bar{v}_{\omega}-\frac{1}{2} p\left((1-\lambda) \bar{v}_{r}+\nabla_{\omega} \cdot \bar{v}_{\omega}\right) \\
& \left.-\frac{1}{2}\left((\lambda+2) u_{r}+\nabla_{\omega} \cdot u_{\omega}\right) \bar{q}\right) d \omega
\end{aligned}
$$

where

$$
\begin{aligned}
& u_{\omega}=\left(\begin{array}{l}
u_{\theta} \\
u_{\varphi}
\end{array}\right), \quad \nabla_{\omega} \cdot u_{\omega}=\frac{1}{\sin \theta} \partial_{\theta}\left(\sin \theta u_{\theta}\right)+\frac{1}{\sin \theta} \partial_{\varphi} u_{\varphi}, \\
& \nabla_{\omega} u_{r}=\left(\begin{array}{c}
\partial_{\theta} u_{r} \\
(\sin \theta)^{-1} \partial_{\varphi} u_{r}
\end{array}\right), \\
& {\left[u_{\omega}, v_{\omega}\right]=\int_{\Omega}\left(\partial_{\theta} u_{\theta} \cdot \partial_{\theta} \bar{v}_{\theta}+\left(\frac{1}{\sin \theta} \partial_{\varphi} u_{\varphi}+\cot \theta u_{\theta}\right) \cdot\left(\frac{1}{\sin \theta} \partial_{\varphi} \bar{v}_{\varphi}+\cot \theta \bar{v}_{\theta}\right)\right.} \\
& \left.+\frac{1}{2}\left(\partial_{\theta} u_{\varphi}+\frac{1}{\sin \theta} \partial_{\varphi} u_{\theta}-\cot \theta u_{\varphi}\right) \cdot\left(\partial_{\theta} \bar{v}_{\varphi}+\frac{1}{\sin \theta} \partial_{\varphi} \bar{v}_{\theta}-\cot \theta \bar{v}_{\varphi}\right)\right) d \omega .
\end{aligned}
$$

One directly verifies that 


$$
\begin{aligned}
{\left[u_{\omega}, u_{\omega}\right] } & =\frac{1}{2} \int_{\Omega}\left|\nabla_{\omega} \cdot u_{\omega}\right|^{2} d \omega+\frac{1}{2} \int_{\Omega}\left|\partial_{\theta} u_{\theta}-\frac{1}{\sin \theta} \partial_{\varphi} u_{\varphi}-\cot \theta u_{\theta}\right|^{2} d \omega \\
& +\frac{1}{2} \int_{\Omega}\left|\partial_{\theta} u_{\varphi}+\frac{1}{\sin \theta} \partial_{\varphi} u_{\theta}-\cot \theta u_{\varphi}\right|^{2} d \omega .
\end{aligned}
$$

Hence

$$
\left[u_{\omega}, u_{\omega}\right] \geq \frac{1}{2} \int_{\Omega}\left|\nabla_{\omega} \cdot u_{\omega}\right|^{2} d \omega .
$$
We denote by $h^{1}(\Omega)$ the set of all vector-functions $u_{\omega}=\left(\begin{array}{l}u_{\theta} \\ u_{\varphi}\end{array}\right)$ such that the
quantity

$$
\begin{aligned}
\left\|u_{\omega}\right\|_{h^{1}(\Omega)} & =\left(\int _ { \Omega } \left(\left|u_{\omega}\right|^{2}+\left|\partial_{\theta} u_{\theta}\right|^{2}+\left|\partial_{\theta} u_{\varphi}\right|^{2}+\left|\frac{1}{\sin \theta} \partial_{\varphi} u_{\theta}-\cot \theta u_{\varphi}\right|^{2}\right.\right. \\
& \left.\left.+\left|\frac{1}{\sin \theta} \partial_{\varphi} u_{\varphi}+\cot \theta u_{\theta}\right|^{2}\right) d \omega\right)^{1 / 2}
\end{aligned}
$$

is finite and by $h^{1}(\Omega)$ the closure of $C_{0}^{\infty}(\Omega)^{2}$ with respect to the norm (2.4). According to Proposition 1.1 in [10], the Cartesian components of the vector-valued function $u=\left(u_{1}, u_{2}, u_{3}\right)$ belong to $H^{1}(\Omega)$ if and only if $u_{r} \in H^{1}(\Omega)$ and $u_{\omega} \in h^{1}(\Omega)$. The following lemma gives an equivalent norm in $h^{1}(\Omega)$.

Lemma 2.1. For all $u_{\omega} \in h^{1}(\Omega)$ the inequality

$$
\left[u_{\omega}, u_{\omega}\right]+\int_{\Omega}\left|u_{\omega}\right|^{2} d \omega \geq c_{0}\left\|u_{\omega}\right\|_{h^{1}(\Omega)}^{2}
$$

holds, where $c_{0}$ is a positive constant.

Proof. Let $\mathscr{K}_{0}$ be the set $\left\{x \in \mathscr{K}: \frac{1}{2}<|x|<2\right\}$. Since the boundary of $\mathscr{K}_{0}$ is Lipschitz, the Korn inequality (see [2])

$$
\int_{\mathscr{K}_{0}}\left(\sum_{i, j=1}^{3}\left|\varepsilon_{i, j}(U)\right|^{2}+|U|^{2}\right) d x \geq c \int_{\mathscr{K}_{0}} \sum_{i, j=1}^{3}\left|\partial_{x_{i}} U_{j}\right|^{2} d x
$$

is valid. Taking $U(x)=u(\omega)$ and writing the last inequality in the spherical components, we obtain

$$
a\left(\left(\begin{array}{l}
u \\
0
\end{array}\right),\left(\begin{array}{l}
u \\
0
\end{array}\right) ; 0\right)+\int_{\Omega}\left(\left|u_{\omega}\right|^{2}+\left|u_{r}\right|^{2}\right) d \omega \geq c\left(\left\|u_{\omega}\right\|_{h^{1}(\Omega)}^{2}+\left\|u_{r}\right\|_{H^{1}(\Omega)}^{2}\right) .
$$

Setting $u_{r}=0$, we arrive at (2.5). 
We define the space $\mathscr{H}_{s}$ as the set of all $\left(u_{r}, u_{\omega}\right) \in H^{1}(\Omega) \times h^{1}(\Omega)$ such that the boundary conditions (i)--(iii) are satisfied, i.e., we have $\left(u_{1}, u_{2}, u_{3}\right) \in \mathscr{H}$ if and only if $\left(u_{r}, u_{\omega}\right) \in \mathscr{H}_{s}$.

The form $a(\cdot, \cdot ; \lambda)$ is continuous on $\left(\mathscr{H}_{s} \times L_{2}(\Omega)\right) \times\left(\mathscr{H}_{s} \times L_{2}(\Omega)\right)$ and generates a continuous operator

$$
\mathfrak{U}(\lambda): \mathscr{H}_{s} \times L_{2}(\Omega) \rightarrow \mathscr{H}_{s}^{*} \times L_{2}(\Omega)
$$

by the equality

$$
\left(\mathfrak{H}(\lambda)\left(\begin{array}{l}
u \\
p
\end{array}\right),\left(\begin{array}{l}
v \\
q
\end{array}\right)\right)_{L_{2}(\Omega)^{4}}=a\left(\left(\begin{array}{l}
u \\
p
\end{array}\right),\left(\begin{array}{l}
v \\
q
\end{array}\right) ; \lambda\right), \quad u, v \in \mathscr{H}_{s}, p, q \in L_{2}(\Omega) .
$$

This is the operator pencil mentioned in the introduction. We shall study its spectrum.

\section{Basic properties of the pencil $\mathfrak{A}$.}

We prove that the operator $\mathfrak{A}(\lambda)$ is Fredholm for every fixed $\lambda$, and invertible for at least one $\lambda$. For the proof of this assertion we need the following two lemmas.

Lemma 3.1. Let $g$ be an arbitrary $L_{2}$ function on $\Omega$. Then the equation

$$
(\lambda+2) u_{r}+\nabla_{\omega} \cdot u_{\omega}=g
$$

has a solution $\left(u_{r}, u_{\omega}\right) \in \stackrel{\circ}{H^{1}}(\Omega) \times \stackrel{\circ}{h^{1}}(\Omega)$ satisfying the inequality

$$
|\lambda+2|\left\|u_{r}\right\|_{H^{1}(\Omega)}+\left\|u_{\omega}\right\|_{h^{1}(\Omega)} \leq c\|g\|_{L_{2}(\Omega)}
$$

with a constant $c$ independent of $g$ and $\lambda$.

Proof. First let $\int_{\Omega} g d \omega=0$. Then according to Lemma 2.3 in [11], the equation $\nabla_{\omega} \cdot u_{\omega}=g$ has a solution $u_{\omega} \in h^{1}(\Omega)$. Thus, the vector-function $\left(0, u_{\omega}\right)$ satisfies the desired equation.

In the case $\int_{\Omega} g d \omega=c \neq 0$ we set $u_{r}=c(\lambda+2)^{-1} \psi$, where $\psi$ is an arbitrary function in $\stackrel{\circ}{H}^{1}(\Omega)$ such that $\int_{\Omega} \psi d \omega=1$. Then the integral of the function $g-(\lambda+2) u_{r}$ over $\Omega$ vanishes, and we can solve the equation $\nabla_{\omega} \cdot u_{\omega}=g-(\lambda+2) u_{r}$. The so obtained vector-function $\left(u_{r}, u_{\omega}\right)$ has the desired properties.

Lemma 3.2. Let $\operatorname{Re} \lambda=-1 / 2$, and let $|\lambda|$ be sufficiently large. Then for every $f \in \mathscr{H}_{s}^{*}, g \in L_{2}(\Omega)$ there exists a vector-function $(u, p)$ satisfying the equality

$$
a\left(\left(\begin{array}{l}
u \\
p
\end{array}\right),\left(\begin{array}{l}
v \\
q
\end{array}\right) ; \lambda\right)=(f, v)_{\Omega}+\int_{\Omega} g \bar{q} d \omega
$$


for all $v \in \mathscr{H}_{s}, q \in L_{2}(\Omega)$. Here and in what follows by $(\cdot, \cdot)_{\Omega}$ we denote the continuation of the scalar product in $L_{2}(\Omega)^{3}$ to $\mathscr{H}_{s}^{*} \times \mathscr{H}_{s}$.

Proof. By Lemma 3.1, there exists a vector-function $u^{(0)}=\left(u_{r}^{(0)}, u_{\omega}^{(0)}\right) \in$ $\mathscr{H}_{s}$ satisfying the equation $(\lambda+2) u_{r}^{(0)}+\nabla_{\omega} \cdot u_{\omega}^{(0)}=-2 g$. Then according to (2.2), we have

$$
a\left(\left(\begin{array}{c}
u^{(0)} \\
0
\end{array}\right),\left(\begin{array}{c}
v \\
q
\end{array}\right) ; \lambda\right)=(F, v)_{\Omega}+\int_{\Omega} g \bar{q} d \omega
$$

where

$$
\begin{aligned}
(F, v)_{\Omega} & =\left[u_{\omega}^{(0)}, v_{\omega}\right]+\int_{\Omega}\left(\frac{1}{2} \nabla_{\omega} u_{r}^{(0)} \cdot \nabla_{\omega} \bar{v}_{r}+(\lambda+2)(1-\lambda) u_{r}^{(0)} \bar{v}_{r}\right. \\
& +\frac{1}{2}(\lambda+2)(1-\lambda) u_{\omega}^{(0)} \cdot \bar{v}_{\omega}+u_{r}^{(0)} \nabla_{\omega} \cdot \bar{v}_{\omega}+\left(\nabla_{\omega} \cdot u_{\omega}^{(0)}\right) \bar{v}_{r} \\
& \left.-\frac{1-\lambda}{2} u_{\omega}^{(0)} \cdot \nabla_{\omega} \bar{v}_{r}-\frac{\lambda+2}{2}\left(\nabla_{\omega} u_{r}^{(0)}\right) \cdot \bar{v}_{\omega}\right) d \omega
\end{aligned}
$$

Obviously, $F$ is a continuous functional on $\mathscr{H}_{s}$.

We show now that there exists a vector-function $\left(u^{(1)}, p\right) \in \mathscr{H}_{s} \times L_{2}(\Omega)$ such that

$$
(\lambda+2) u_{r}^{(1)}+\nabla_{\omega} \cdot u_{\omega}^{(1)}=0
$$

and

$$
a\left(\left(\begin{array}{c}
u^{(1)} \\
p
\end{array}\right),\left(\begin{array}{l}
v \\
q
\end{array}\right) ; \lambda\right)=(f-F, v)_{\Omega}
$$

for $v \in \mathscr{H}_{s}, q \in L_{2}(\Omega)$. Then the vector-function $(u, p)$ with $u=u^{(0)}+u^{(1)}$ satisfies (3.1).

For $\operatorname{Re} \lambda=-1 / 2$ we have

$$
\begin{aligned}
a\left(\left(\begin{array}{c}
u \\
0
\end{array}\right),\left(\begin{array}{c}
u \\
0
\end{array}\right) ; \lambda\right) & =\int_{\Omega}\left(|\lambda|^{2}\left|u_{r}\right|^{2}+\left|\partial_{\theta} u_{\theta}+u_{r}\right|^{2}+\left|\frac{1}{\sin \theta} \partial_{\varphi} u_{\varphi}+u_{\theta} \cot \theta+u_{r}\right|^{2}\right. \\
& +\frac{1}{2}\left|\partial_{\theta} u_{\varphi}+\frac{1}{\sin \theta} \partial_{\varphi} u_{\theta}-u_{\varphi} \cot \theta\right|^{2}+\frac{1}{2}\left|(\lambda-1) u_{\theta}+\partial_{\theta} u_{r}\right|^{2} \\
& \left.+\frac{1}{2}\left|(\lambda-1) u_{\varphi}+\frac{1}{\sin \theta} \partial_{\varphi} u_{r}\right|^{2}\right) d \omega
\end{aligned}
$$

Since this form vanishes only for $u=0$, it follows from Lemma 2.1 that 


$$
\left|a\left(\left(\begin{array}{c}
u \\
0
\end{array}\right),\left(\begin{array}{l}
u \\
0
\end{array}\right) ; \lambda\right)\right| \geq c\|u\|_{\mathscr{H}_{s}}^{2},
$$

if $|\lambda|$ is sufficiently large, where the constant $c$ depends on $\lambda$ but not on $u$. In particular, (3.2) is satisfied for all $u \in \mathscr{H}_{s}(\lambda) \stackrel{\text { def }}{=}\left\{u \in \mathscr{H}_{s}:(\lambda+2) u_{r}+\right.$ $\left.\nabla_{\omega} \cdot u_{\omega}=0\right\}$. Consequently, by Lax-Milgram's theorem, there exists a solution $u^{(1)} \in \mathscr{H}_{s}^{(\lambda)}$ of the equation

$$
a\left(\left(\begin{array}{c}
u^{(1)} \\
0
\end{array}\right),\left(\begin{array}{c}
v \\
0
\end{array}\right) ; \lambda\right)=(f-F, v)_{\Omega}, \quad v \in \mathscr{H}_{s}^{(\lambda)} .
$$

We consider the functional

$$
v \rightarrow G(v) \stackrel{\operatorname{def}}{=} a\left(\left(\begin{array}{c}
u^{(1)} \\
0
\end{array}\right),\left(\begin{array}{l}
v \\
0
\end{array}\right) ; \lambda\right)-(f-F, v)_{\Omega}
$$

on $\mathscr{H}_{s}$. By (3.3), the functional $G$ vanishes on the subspace $\mathscr{H}_{s}(\lambda)$ of $\mathscr{H}_{s}$. Hence we have

$$
|G(v)|=\min |G(w)| \leq c\|w\|_{\mathscr{H}_{s}},
$$

where the minimum is taken over all $w \in \mathscr{H}_{s}$ such that $(\lambda+2) w_{r}+\nabla_{\omega} \cdot w_{\omega} \bar{o}(\lambda+2) v_{r}+\nabla_{\omega} \cdot v_{\omega}$. Due to Lemma 3.1, we can choose $w \in H^{1}(\Omega) \times h^{1}(\Omega)$ such that

$$
\|w\|_{\mathscr{H}_{s}} \leq c\left\|(\lambda+2) v_{r}+\nabla_{\omega} \cdot v_{\omega}\right\|_{L_{2}(\Omega)} .
$$

Therefore, $G(v)$ can be understood as a linear and continuous functional applied to $(\lambda+2) v_{r}+\nabla_{\omega} \cdot v_{\omega}$. By the Riesz theorem, there exists a function $p \in L_{2}(\Omega)$ such that

$$
G(v)=\frac{1}{2} \int_{\Omega} p \cdot\left((\lambda+2) \bar{v}_{r}+\nabla_{\omega} \cdot \bar{v}_{\omega}\right) d \omega
$$

for all $v \in \mathscr{H}_{s}$. Using (3.6), we obtain

$$
\begin{aligned}
& a\left(\left(\begin{array}{c}
u^{(1)} \\
p
\end{array}\right),\left(\begin{array}{c}
v \\
q
\end{array}\right) ; \lambda\right)=a\left(\left(\begin{array}{c}
u^{(1)} \\
0
\end{array}\right),\left(\begin{array}{c}
v \\
0
\end{array}\right) ; \lambda\right) \\
& -\frac{1}{2} \int_{\Omega} p \cdot\left((\lambda+2) \bar{v}_{r}+\nabla_{\omega} \cdot \bar{v}_{\omega}\right) d \omega=(f-F, v)_{\Omega} .
\end{aligned}
$$

Thus, the vector-function $\left(u^{(1)}, p\right)$ satisfies the desired equation. This proves the lemma. 
ThEOREM 3.1. 1) The operator $\mathfrak{A}(\lambda)$ is Fredholm for all $\lambda$.

2) The spectrum of the pencil $\mathfrak{A}(\lambda)$ consists of isolated eigenvalues with finite algebraic multiplicities.

3) The line $\operatorname{Re} \lambda=-1 / 2$ does not contain eigenvalues of the pencil $\mathfrak{A}(\lambda)$.

4) The number $\lambda_{0}$ is an eigenvalue of the pencil $\mathfrak{A}(\lambda)$ if and only if $-1-\bar{\lambda}_{0}$ is an eigenvalue of this pencil. The geometric, algebraic and partial multiplicities of the eigenvalues $\lambda_{0}$ and $-1-\bar{\lambda}_{0}$ coincide.

Proof. 1) We show first that the kernel of the operator $\mathfrak{A}(\lambda)$ is trivial for $\operatorname{Re} \lambda=-1 / 2$. Let $(u, p)$ be an element of the kernel of $\mathfrak{A}(\lambda)$, where $\lambda$ is a number on the line $\operatorname{Re} \lambda=-1 / 2$. Then

$$
0=a\left(\left(\begin{array}{l}
u \\
p
\end{array}\right),\left(\begin{array}{l}
0 \\
q
\end{array}\right) ; \lambda\right)=-\frac{1}{2} \int_{\Omega}\left((\lambda+2) u_{r}+\nabla_{\omega} \cdot u_{\omega}\right) \bar{q} d \omega
$$

for all $q \in L_{2}(\Omega)$. This implies

$$
(\lambda+2) u_{r}+\nabla_{\omega} \cdot u_{\omega}=0 \quad \text { in } \Omega .
$$

Using (3.5) and the equality $\bar{\lambda}+2=1-\lambda$, we obtain

$$
\begin{aligned}
0 & =a\left(\left(\begin{array}{c}
u \\
p
\end{array}\right),\left(\begin{array}{c}
u \\
p
\end{array}\right) ; \lambda\right)=\left[u_{\omega}, u_{\omega}\right]-\frac{1}{2} \int_{\Omega}\left|\nabla_{\omega} \cdot u_{\omega}\right|^{2} d \omega \\
& +\int_{\Omega}\left(\frac{1}{2}\left|\nabla_{\omega} u_{r}-(1-\lambda) u_{\omega}\right|^{2}+\frac{1}{2}\left|\nabla_{\omega} \cdot u_{\omega}+2 u_{r}\right|^{2}+\left(|1-\lambda|^{2}-2\right)\left|u_{r}\right|^{2}\right) d \omega .
\end{aligned}
$$

Since $2\left[u_{\omega}, u_{\omega}\right] \geq \int_{\Omega}\left|\nabla_{\omega} \cdot u_{\omega}\right|^{2} d \omega$ and $|1-\lambda|^{2} \geq 9 / 4$, the last equation is satisfied only, if $u_{r}=0$ and $u_{\omega}=0$. Furthermore, for $u=0$ we get

$$
a\left(\left(\begin{array}{c}
u \\
p
\end{array}\right),\left(\begin{array}{c}
v \\
0
\end{array}\right) ; \lambda\right)=-\frac{1}{2} \int_{\Omega} p\left((1-\lambda) v_{r}+\nabla_{\omega} \cdot v_{\omega}\right) d \omega=0, \quad\left(v_{r}, v_{\omega}\right) \in \mathscr{H}_{s} .
$$

In particular, the last equation is satisfied for all $v_{r} \in \stackrel{\circ}{H}^{1}(\Omega), v_{\omega}=0$. From this we conclude $p=0$. Thus, the kernel of $\mathfrak{A}(\lambda)$ is trivial for $\operatorname{Re} \lambda=-1 / 2$.

If moreover $|\lambda|$ is sufficiently large, then by Lemma 3.2, the operator $\mathfrak{A}(\lambda)$ is an isomorphism. Since $\mathfrak{A}(\lambda)-\mathfrak{U}(\mu)$ is a compact operator for arbitrary $\lambda$, $\mu$, it follows that the operator $\mathfrak{A}(\lambda)$ is Fredholm for every complex $\lambda$. This proves assertion 1). 
2) The second assertion follows from 1) and from the invertibility of $\mathfrak{A}(\lambda)$ for $\lambda=-1 / 2$ (see e.g. [3]).

3) The proof of the third assertion is contained in the proof of assertion 1).

4) It can be easily seen that

$$
a\left(\left(\begin{array}{l}
u \\
p
\end{array}\right),\left(\begin{array}{l}
v \\
q
\end{array}\right) ; \lambda\right)=\overline{a\left(\left(\begin{array}{l}
v \\
q
\end{array}\right),\left(\begin{array}{l}
u \\
p
\end{array}\right) ;-1-\bar{\lambda}\right)},
$$

i.e., $\mathfrak{A}(\lambda)^{*}=\mathfrak{A}(-1-\bar{\lambda})$. As a consequence of this equality, assertion 4) holds.

In a standard way it can be shown that the integral identity (1.5) has a solution of the form (1.6), (1.7) if and only if $\lambda_{0}$ is an eigenvalue of the pencil $\mathfrak{A}(\lambda)$ and the vector-functions $\left(u^{(0)}, p^{(0)}\right), \ldots,\left(u^{(s)}, p^{(s)}\right)$ form a Jordan chain to this eigenvalue, i.e., $\left(u^{(0)}, p^{(0)}\right)$ is an eigenvector to $\lambda_{0}$ and $\left(u^{(1)}, p^{(1)}\right), \ldots$, $\left(u^{(s)}, p^{(s)}\right)$ are generalized eigenvectors associated with $\left(u^{(0)}, p^{(0)}\right)$.

In the sequel the following properties of the spaces $\mathscr{H}$ and $\mathscr{H}_{s}$ will play an important role.

Lemma 3.3. 1) The subspace $\mathscr{H}_{s}$ admits the representation

$$
\mathscr{H}_{s}=\mathscr{H}_{s}^{r} \times \mathscr{H}_{s}^{\omega},
$$

where $\mathscr{H}_{s}^{r}$ is a subspace of $H^{1}(\Omega), \stackrel{\circ}{H}^{1}(\Omega) \subset \mathscr{H}_{s}^{r}$, and $\mathscr{H}_{s}^{\omega}$ is a subspace of $h^{1}(\Omega), h^{1}(\Omega) \subset \mathscr{H}_{s}^{\omega}$.

2) For all $u, v \in \mathscr{H}$ the equality

$$
\int_{\partial \Omega} u_{n} \bar{v}_{r} d \omega^{\prime}=0
$$

or, equivalently,

$$
\int_{\Omega}\left(\left(\nabla_{\omega} \cdot u_{\omega}\right) \bar{v}_{r}+u_{\omega} \cdot \nabla_{\omega} \bar{v}_{r}\right) d \omega=0
$$

is valid. 
Proof. 1) In order to prove (3.7) we have to show that $\left(u_{r}, u_{\omega}\right) \in \mathscr{H}_{s}$ implies $\left(u_{r}, 0\right) \in \mathscr{H}_{s}$.

Let $\left(u_{r}, u_{\omega}\right)$ be an element of $\mathscr{H}_{s}$. The Cartesian components of the vectorfunction $\left(u_{r}, 0\right)$ are

$$
w=\left(\begin{array}{l}
w_{1} \\
w_{2} \\
w_{3}
\end{array}\right)=J^{*}\left(\begin{array}{c}
u_{r} \\
0
\end{array}\right)=\left(\begin{array}{c}
\sin \theta \cos \varphi \\
\sin \theta \sin \varphi \\
\cos \theta
\end{array}\right) u_{r} .
$$

If $k \in I_{0} \cup I_{\tau}$, then $u_{r}=0$ on $\gamma_{k}$ and, therefore, $w=0$ on $\gamma_{k}$. Since the vector $(\sin \theta \cos \varphi, \sin \theta \sin \varphi, \cos \theta)=x /|x|$ is orthogonal to $n$, we further have $w_{n}=0$ on $\gamma_{k}$ for every $k=1, \ldots, N$. Thus, $w \in \mathscr{H}$ and, consequently, $\left(u_{r}, 0\right) \in \mathscr{H}_{s}$.

2) If $k \in I_{0} \cup I_{\tau}$, then $v_{r}=0$ on $\gamma_{k}$, while $u_{n}=0$ on $\gamma_{k}$ for $k \in I_{n}$. Hence

$$
\int_{\gamma_{k}} u_{n} \cdot \bar{v}_{r} d \omega^{\prime}=0
$$

for $k=1, \ldots, N$. This implies (3.8).

It remains to prove that the left sides of (3.8) and (3.9) coincide. Using the representation of $u_{\omega}$ by the Cartesian components of $u$, we get

$$
\begin{aligned}
& \int_{\Omega}\left(\left(\nabla_{\omega} \cdot u_{\omega}\right) \bar{v}_{r}+u_{\omega} \cdot \nabla_{\omega} \bar{v}_{r}\right) d \omega \\
& =\int_{\Omega}(\sin \theta)^{-1}\left(\partial_{\theta}\left(\sin \theta u_{\theta} \bar{v}_{r}\right)+\partial_{\varphi}\left(u_{\varphi} \bar{v}_{r}\right)\right) d \omega \\
& =\int_{\Omega}\left(\cos \theta \cos \varphi \partial_{\theta}\left(u_{1} \bar{v}_{r}\right)+\cos \theta \sin \varphi \partial_{\theta}\left(u_{2} \bar{v}_{r}\right)\right. \\
& -\sin \theta \partial_{\theta}\left(u_{3} \bar{v}_{r}\right)-\frac{\sin \varphi}{\sin \theta} \partial_{\varphi}\left(u_{1} \bar{v}_{r}\right) \\
& +\frac{\cos \varphi}{\sin \theta} \partial_{\varphi}\left(u_{2} \bar{v}_{r}\right)-2 \sin \theta \cos \varphi u_{1} \bar{v}_{r} \\
& \left.-2 \sin \theta \sin \varphi u_{2} \bar{v}_{r}-2 \cos \theta u_{3} \bar{v}_{r}\right) d \omega .
\end{aligned}
$$


Integrating by parts, we further obtain

$$
\begin{aligned}
& \frac{1}{\log 2} \int_{\substack{\mathscr{S} \\
1<|x|<2}} \nabla_{x} \cdot\left(r^{-2} u(\omega) \overline{v_{r}(\omega)}\right) d x \\
& =\frac{1}{\log 2} \int_{\substack{\partial \mathscr{K} \\
1<|x|<2}} n \cdot r^{-2} u(\omega) \overline{v_{r}(\omega)} d x^{\prime}=\int_{\partial \Omega} u_{n} \bar{v}_{r} d \omega^{\prime} .
\end{aligned}
$$

The integrand on the right side of (3.11) is equal to

$$
\begin{aligned}
& \nabla_{x} \cdot\left(r^{-2} u(\omega) \overline{v_{r}(\omega)}\right) \\
& =\frac{1}{r^{3}}\left(\cos \theta \cos \varphi \partial_{\theta}\left(u_{1} \bar{v}_{r}\right)+\cos \theta \sin \varphi \partial_{\theta}\left(u_{2} \bar{v}_{r}\right)\right. \\
& -\sin \theta \partial_{\theta}\left(u_{3} \bar{v}_{r}\right)-\frac{\sin \varphi}{\sin \theta} \partial_{\varphi}\left(u_{1} \bar{v}_{r}\right) \\
& \left.+\frac{\cos \varphi}{\sin \theta} \partial_{\varphi}\left(u_{2} \bar{v}_{r}\right)-2 \sin \theta \cos \varphi u_{1} \bar{v}_{r}-2 \sin \theta \sin \varphi u_{2} \bar{v}_{r}-2 \cos \theta u_{3} \bar{v}_{r}\right) .
\end{aligned}
$$

Hence the integral on the left side of (3.11) coincides with the expression (3.10). The lemma is proved.

REMARK 3.1. In the following we will only use the properties (3.7) and (3.8) of the space $\mathscr{H}$. All results of this paper are also true for other subspaces $\mathscr{H} \subset H^{1}(\Omega) \times h^{1}(\Omega)$ satisfying these conditions.

Due to (3.8) the sesquilinear form $a(\cdot, \cdot ; \lambda)$ can be written on $\left(\mathscr{H}_{s} \times L_{2}(\Omega)\right)^{2}$ in the form

$$
\begin{aligned}
& a\left(\left(\begin{array}{l}
u \\
p
\end{array}\right),\left(\begin{array}{l}
v \\
q
\end{array}\right) ; \lambda\right)=\left[u_{\omega}, v_{\omega}\right] \\
& +\int_{\Omega}\left(\frac{1}{2} \nabla_{\omega} u_{r} \cdot \nabla_{\omega} \bar{v}_{r}+(\lambda+2)(1-\lambda)\left(u_{r} \bar{v}_{r}+\frac{1}{2} u_{\omega} \cdot \bar{v}_{\omega}\right)\right. \\
& +\left(\frac{\lambda+2}{2}+1\right) u_{r} \nabla_{\omega} \cdot \bar{v}_{\omega}+\left(\frac{1-\lambda}{2}+1\right)\left(\nabla_{\omega} \cdot u_{\omega}\right) \bar{v}_{r} \\
& \left.-\frac{1}{2} p\left((1-\lambda) \bar{v}_{r}+\nabla_{\omega} \cdot \bar{v}_{\omega}\right)-\frac{1}{2}\left((\lambda+2) u_{r}+\nabla_{\omega} \cdot u_{\omega}\right) \bar{q}\right) d \omega .
\end{aligned}
$$


We introduce the matrix

$$
\mathcal{S}(\lambda)=\left(\begin{array}{cccc}
\lambda+2 & 0 & 0 & 0 \\
0 & 1-\lambda & 0 & 0 \\
0 & 0 & 1-\lambda & 0 \\
2+4 \lambda & 0 & 0 & 1-\lambda
\end{array}\right)
$$

According to (3.7) the vector-function $\left((\lambda+2) u_{r},(1-\lambda) u_{\omega}\right)$ belongs to $\mathscr{H}_{s}$, if $\left(u_{r}, u_{\omega}\right) \in \mathscr{H}_{s}$. Moreover, we have

$$
a\left(\left(\begin{array}{l}
u \\
p
\end{array}\right), \mathcal{S}(\bar{\lambda})\left(\begin{array}{l}
v \\
q
\end{array}\right) ; \lambda\right)=a\left(\mathcal{S}(\lambda)\left(\begin{array}{l}
u \\
p
\end{array}\right),\left(\begin{array}{l}
v \\
q
\end{array}\right) ;-1-\lambda\right)
$$

for all $u, v \in \mathscr{H}_{s}, p, q \in L_{2}(\Omega)$. Consequently, there is the equality

$$
\mathcal{S}(\lambda)^{t} \mathfrak{A}(\lambda)=\mathfrak{A}(-1-\lambda) \mathcal{S}(\lambda), \quad \lambda \in \mathbf{C},
$$

where $\mathcal{S}(\lambda)^{t}$ is the transposed matrix to $\mathcal{S}(\lambda)$. This leads to the following assertions.

THEOREM 3.2. Let $\lambda_{0}$ be an eigenvalue of the pencil $\mathfrak{A}(\lambda)$ and let $\left(u^{(0)}, p^{(0)}\right), \ldots\left(u^{(s)}, p^{(s)}\right)$ be a Jordan chain to this eigenvalue.

1) If $\lambda_{0} \notin\{1,-2\}$ or $\lambda_{0} \in\{1,-2\}$ and $\mathcal{S}\left(\lambda_{0}\right)\left(\begin{array}{c}u^{(0)} \\ p^{(0)}\end{array}\right) \neq 0$, then $-1-\lambda_{0}$ is also an eigenvalue of the pencil $\mathfrak{A}(\lambda)$ and the vector-functions $\left(\begin{array}{c}v^{(0)} \\ q^{(0)}\end{array}\right)=S\left(\lambda_{0}\right)\left(\begin{array}{c}u^{(0)} \\ p^{(0)}\end{array}\right)$,

$$
\left(\begin{array}{l}
v^{(k)} \\
q^{(k)}
\end{array}\right)=(-1)^{k}\left(\mathcal{S}\left(\lambda_{0}\right)\left(\begin{array}{l}
u^{(k)} \\
p^{(k)}
\end{array}\right)+\left(\begin{array}{cccc}
1 & 0 & 0 & 0 \\
0 & -1 & 0 & 0 \\
0 & 0 & -1 & 0 \\
4 & 0 & 0 & -1
\end{array}\right)\left(\begin{array}{l}
u^{(k-1)} \\
p^{(k-1)}
\end{array}\right)\right)
$$

$k=1, \ldots, s$, form a Jordan chain to $-1-\lambda_{0}$.

2) If $\lambda_{0} \in\{1,-2\}, \mathcal{S}\left(\lambda_{0}\right)\left(\begin{array}{c}u^{(0)} \\ p^{(0)}\end{array}\right)=0$ and $s \geq 1$, then $-1-\lambda_{0}$ is also an eigenvalue and the vectors

$$
\left(\begin{array}{l}
v^{(k)} \\
q^{(k)}
\end{array}\right)=(-1)^{k}\left(\mathcal{S}\left(\lambda_{0}\right)\left(\begin{array}{l}
u^{(k)} \\
p^{(k)}
\end{array}\right)+\left(\begin{array}{cccc}
1 & 0 & 0 & 0 \\
0 & -1 & 0 & 0 \\
0 & 0 & -1 & 0 \\
4 & 0 & 0 & -1
\end{array}\right)\left(\begin{array}{l}
u^{(k-1)} \\
p^{(k-1)}
\end{array}\right)\right)
$$


$k=1, \ldots, s$, form a Jordan chain to $-1-\lambda_{0}$.

Proof. First note that

$$
\mathcal{S}\left(\lambda_{0}\right)\left(\begin{array}{l}
u^{(1)} \\
p^{(1)}
\end{array}\right)+\left(\begin{array}{cccc}
1 & 0 & 0 & 0 \\
0 & -1 & 0 & 0 \\
0 & 0 & -1 & 0 \\
4 & 0 & 0 & -1
\end{array}\right)\left(\begin{array}{l}
u^{(0)} \\
p^{(0)}
\end{array}\right) \neq 0,
$$

if $\lambda_{0} \in\{1,-2\}$ and $\mathcal{S}\left(\lambda_{0}\right)\left(\begin{array}{c}u^{(0)} \\ p^{(0)}\end{array}\right)=0$.

By the assumptions of the lemma, the vector-functions $\left(u^{(j)}, p^{(j)}\right)$ satisfy the equations

$$
\sum_{j=0}^{k} \frac{1}{(k-j) !} \mathfrak{A}^{(k-j)}\left(\lambda_{0}\right)\left(\begin{array}{l}
u^{(j)} \\
p^{(j)}
\end{array}\right)=0 \quad \text { for } k=0,1, \ldots, s,
$$

where $\mathfrak{P}^{(j)}(\lambda)=d^{j} \mathfrak{X}(\lambda) / d \lambda^{j}$. We have to show that

$$
\begin{aligned}
& \sum_{j=0}^{k} \frac{1}{(k-j) !} \mathfrak{A}^{(k-j)}\left(-1-\lambda_{0}\right)\left(\begin{array}{c}
v^{(j)} \\
q^{(j)}
\end{array}\right) \\
& =(-1)^{k} \mathfrak{U}\left(-1-\lambda_{0}\right) \mathcal{S}\left(\lambda_{0}\right)\left(\begin{array}{c}
u^{(k)} \\
p^{(k)}
\end{array}\right) \\
& +\sum_{j=0}^{k-1} \frac{(-1)^{j}}{(k-j) !}\left(\mathfrak{U}\left(-1-\lambda_{0}\right) \mathcal{S}\left(\lambda_{0}\right)\right. \\
& \left.-(k-j) \mathfrak{I}^{(k-j-1)}\left(-1-\lambda_{0}\right) \mathcal{S}^{\prime}\left(\lambda_{0}\right)\right)\left(\begin{array}{c}
u^{(j)} \\
p^{(j)}
\end{array}\right)=0 .
\end{aligned}
$$

Differentiating (3.13) with respect to $\lambda$, we obtain

$$
\begin{aligned}
& \mathcal{S}\left(\lambda_{0}\right)^{t} \mathfrak{U}^{(k-j)}(\lambda)+(k-j) \mathcal{S}^{\prime}(\lambda)^{t} \mathfrak{A}^{(k-j-1)}(\lambda) \\
& =(-1)^{k-j}\left(\mathfrak{I}^{(k-j)}(-1-\lambda) \mathcal{S}(\lambda)-(k-j) \mathfrak{U}^{(k-j-1)}(-1-\lambda) \mathcal{S}^{\prime}(\lambda)\right)
\end{aligned}
$$

for $j \leq k-1$. Hence the left side of (3.14) is equal to

$$
\begin{aligned}
& (-1)^{k} \mathcal{S}\left(\lambda_{0}\right)^{t} \sum_{j=0}^{k} \frac{1}{(k-j) !} \mathfrak{A}^{(k-j)}\left(\lambda_{0}\right)\left(\begin{array}{c}
u^{(j)} \\
p^{(j)}
\end{array}\right) \\
& +(-1)^{k} \mathcal{S}^{\prime}\left(\lambda_{0}\right)^{t} \sum_{j=0}^{k-1} \frac{1}{(k-1-j) !} \mathfrak{A}^{(k-1-j)}\left(\lambda_{0}\right)\left(\begin{array}{c}
u^{(j)} \\
p^{(j)}
\end{array}\right)=0 .
\end{aligned}
$$


The theorem is proved.

\section{On real eigenvalues of the pencil $\mathfrak{A}$.}

The sesquilinear form (3.12) is not symmetric in its arguments. In order to obtain a symmetric form, we introduce the matrix

$$
J_{\lambda}=\left(\begin{array}{cccc}
\bar{c} & 0 & 0 & 0 \\
0 & 1 & 0 & 0 \\
0 & 0 & 1 & 0 \\
2 \bar{c}-2 & 0 & 0 & 1
\end{array}\right), \quad c=\frac{\bar{\lambda}+2}{1-\lambda}
$$

Then the sesquilinear form

$$
\begin{aligned}
& s\left(\left(\begin{array}{l}
u \\
p
\end{array}\right),\left(\begin{array}{l}
v \\
q
\end{array}\right) ; \lambda\right) \stackrel{\text { def }}{=} a\left(\left(\begin{array}{l}
u \\
p
\end{array}\right), J_{\lambda}\left(\begin{array}{l}
v \\
q
\end{array}\right) ; \lambda\right) \\
& =\left[u_{\omega}, v_{\omega}\right]+\int_{\Omega}\left(\frac{c}{2}\left(\nabla_{\omega} u_{r}\right) \cdot \nabla_{\omega} \bar{v}_{r}+(\lambda+2)(1-c \lambda) u_{r} \bar{v}_{r}\right. \\
& +\frac{1}{2}(\lambda+2)(1-\lambda) u_{\omega} \cdot \bar{v}_{\omega}+\left(\frac{\lambda+2}{2}+1\right) u_{r} \nabla_{\omega} \cdot \bar{v}_{\omega} \\
& +\left(\frac{\bar{\lambda}+2}{2}+1\right)\left(\nabla_{\omega} \cdot u_{\omega}\right) \bar{v}_{r} \\
& \left.-\frac{1}{2} p\left((\bar{\lambda}+2) \bar{v}_{r}+\nabla_{\omega} \cdot \bar{v}_{\omega}\right)-\frac{1}{2}\left((\lambda+2) u_{r}+\nabla_{\omega} \cdot u_{\omega}\right) \bar{q}\right) d \omega
\end{aligned}
$$

is obviously symmetric.

THEOREM 4.1, The strip

$$
\left|\operatorname{Re} \lambda+\frac{1}{2}\right| \leq \frac{3}{2}
$$

contains only real eigenvalues of the pencil $\mathfrak{U}(\lambda)$.

Proof. Let $\lambda$ be a complex eigenvalue in the strip (4.2) such that $\operatorname{Re} \lambda \neq-1 / 2, \operatorname{Im} \lambda \neq 0$ and let $(u, p)$ be an eigenvector corresponding to this eigenvalue. Using (3.5), we obtain

$$
\begin{aligned}
0=s\left(\left(\begin{array}{c}
u \\
p
\end{array}\right),\left(\begin{array}{l}
u \\
p
\end{array}\right) ; \lambda\right) & =\left[u_{\omega}, u_{\omega}\right]+\int_{\Omega}\left(\frac{\bar{\lambda}+2}{2(1-\lambda)}\left|\nabla_{\omega} u_{r}\right|^{2}-3 \frac{\bar{\lambda}+2}{1-\lambda}\left|u_{r}\right|^{2}\right. \\
& \left.+\frac{1}{2}(\lambda+2)(1-\lambda)\left|u_{\omega}\right|^{2}\right) d \omega .
\end{aligned}
$$


Since

$$
\begin{aligned}
\operatorname{Im} s\left(\left(\begin{array}{l}
u \\
p
\end{array}\right),\left(\begin{array}{l}
u \\
p
\end{array}\right) ; \lambda\right) & =\frac{1}{2} \operatorname{Im} \lambda(2 \operatorname{Re} \lambda+1) \int_{\Omega}\left(\frac{1}{|1-\lambda|^{2}}\left|\nabla_{\omega} u_{r}\right|^{2}\right. \\
& \left.-\frac{6}{|1-\lambda|^{2}}\left|u_{r}\right|^{2}-\left|u_{\omega}\right|^{2}\right) d \omega \\
\operatorname{Re} s\left(\left(\begin{array}{c}
u \\
p
\end{array}\right),\left(\begin{array}{c}
u \\
p
\end{array}\right) ; \lambda\right)= & {\left[u_{\omega}, u_{\omega}\right] } \\
+ & \int_{\Omega}\left(\frac{(1-\operatorname{Re} \lambda)(2+\operatorname{Re} \lambda)+(\operatorname{Im} \lambda)^{2}}{2|1-\lambda|^{2}}\left|\nabla_{\omega} u_{r}\right|^{2}\right. \\
& -3 \frac{(1-\operatorname{Re} \lambda)(2+\operatorname{Re} \lambda)+(\operatorname{Im} \lambda)^{2}}{|1-\lambda|^{2}}\left|u_{r}\right|^{2} \\
& \left.+\frac{1}{2}\left((1-\operatorname{Re} \lambda)(2+\operatorname{Re} \lambda)+(\operatorname{Im} \lambda)^{2}\right)\left|u_{\omega}\right|^{2}\right) d \omega
\end{aligned}
$$

we get

$$
\begin{aligned}
\operatorname{Re} s\left(\left(\begin{array}{c}
u \\
p
\end{array}\right),\left(\begin{array}{c}
u \\
p
\end{array}\right) ; \lambda\right) & -\frac{(1-\operatorname{Re} \lambda)(2+\operatorname{Re} \lambda)+(\operatorname{Im} \lambda)^{2}}{\operatorname{Im} \lambda(2 \operatorname{Re} \lambda+1)} \operatorname{Im} s\left(\left(\begin{array}{c}
u \\
p
\end{array}\right),\left(\begin{array}{c}
u \\
p
\end{array}\right) ; \lambda\right) \\
& =\left[u_{\omega}, u_{\omega}\right]+\left((1-\operatorname{Re} \lambda)(2+\operatorname{Re} \lambda)+(\operatorname{Im} \lambda)^{2}\right) \int_{\Omega}\left|u_{\omega}\right|^{2} d \omega .
\end{aligned}
$$

If $\lambda$ satisfies the inequality (4.2), then the right side of the last equation may be zero only in the case $u_{\omega}=0$. Then from (3.5) it follows that $u_{r}=0$. Hence the form (3.12) is zero only if

$$
\int_{\Omega} p\left((1-\lambda) \bar{v}_{r}+\nabla_{\omega} \cdot \bar{v}_{\omega}\right) d \omega=0 \quad \text { for all } v \in \mathscr{H}_{s} .
$$

The last equality implies

$$
\int_{\Omega} p \cdot \bar{v}_{r} d \omega=0 \quad \text { for all } v_{r} \in \stackrel{\circ}{H}^{1}(\Omega)
$$

and, consequently, $p=0$. Thus, the theorem is proved for the case $\operatorname{Re} \lambda \neq-1 / 2$. For $\operatorname{Re} \lambda=-1 / 2$ the assertion of the theorem follows from the third part of Theorem 3.1. 


\section{On generalized eigenvectors.}

By Theorem 4.1 the eigenvalues in the $-2 \leq \operatorname{Re} \lambda \leq 1$ are real. Then the quantity $c$ in the definition of the matrix $J_{\lambda}$ is equal to $(\lambda+2) /(1-\lambda)$. In this section we will use this expression for the definition of the matrix $J_{\lambda}$.

Lemma 5.1. If $\lambda_{0}$ is a real eigenvalue of the pencil $\mathfrak{A}(\lambda)$ in the interval $(-1 / 2,1)$ and $\left(u^{(0)}, p^{(0)}\right)$ is an eigenvector corresponding to this eigenvalue, then

$$
\left.\frac{d}{d \lambda} a\left(\left(\begin{array}{c}
u^{(0)} \\
p^{(0)}
\end{array}\right), J_{\lambda}\left(\begin{array}{c}
u^{(0)} \\
p^{(0)}
\end{array}\right) ; \lambda\right)\right|_{\lambda=\lambda_{0}}<0
$$

Proof. The left side of (5.1) is equal to

$$
\begin{aligned}
& \left.\frac{d}{d \lambda} s\left(\left(\begin{array}{c}
u^{(0)} \\
p^{(0)}
\end{array}\right),\left(\begin{array}{c}
u^{(0)} \\
p^{(0)}
\end{array}\right) ; \lambda\right)\right|_{\lambda=\lambda_{0}}=\int_{\Omega}\left(\frac{3}{2\left(1-\lambda_{0}\right)^{2}}\left|\nabla_{\omega} u_{r}^{(0)}\right|^{2}\right. \\
& +\left(2 \lambda_{0}+5-\frac{\lambda_{0}+2}{1-\lambda_{0}}-3 \frac{\lambda_{0}+2}{\left(1-\lambda_{0}\right)^{2}}\right)\left|u_{r}^{(0)}\right|^{2}-\frac{1}{2}\left(2 \lambda_{0}+1\right)\left|u_{\omega}^{(0)}\right|^{2}+ \\
& \left.\frac{1}{2} u_{r}^{(0)} \nabla_{\omega} \cdot \overline{u_{\omega}^{(0)}}+\frac{1}{2}\left(\nabla_{\omega} \cdot u_{\omega}^{(0)}\right) \overline{u_{r}^{(0)}}-\frac{1}{2} p \overline{u_{r}^{(0)}}-\frac{1}{2} u_{r}^{(0)} \bar{p}\right) d \omega .
\end{aligned}
$$

Further, since $\left(u^{(0)}, p^{(0)}\right)$ is an eigenvector, we have

$$
a\left(\left(\begin{array}{c}
u^{(0)} \\
p^{(0)}
\end{array}\right),\left(\begin{array}{l}
v \\
q
\end{array}\right) ; \lambda_{0}\right)=0 \quad \text { for all } v \in \mathscr{H}_{s}, q \in L_{2}(\Omega) .
$$

Inserting $v=0$ into (5.3), we get

$$
\left(\lambda_{0}+2\right) u_{r}^{(0)}+\nabla_{\omega} \cdot u_{\omega}^{(0)}=0 \quad \text { in } \Omega .
$$

Furthermore, substituting $v=\left(u_{r}^{(0)}, 0\right), q=0$ into (5.3), we arrive at the equality

$$
\begin{aligned}
& \int_{\Omega}\left(\frac{1}{2}\left|\nabla_{\omega} u_{r}^{(0)}\right|^{2}+\left(\lambda_{0}+2\right)\left(1-\lambda_{0}\right)\left|u_{r}^{(0)}\right|^{2}\right. \\
& \left.+\left(\frac{1-\lambda_{0}}{2}+1\right)\left(\nabla_{\omega} \cdot u_{\omega}^{(0)}\right) \overline{u_{r}^{(0)}}-\frac{1-\lambda_{0}}{2} p^{(0)} \overline{u_{r}^{(0)}}\right) d \omega=0 .
\end{aligned}
$$

Multiplying the real part of the left side of (5.5) by $2\left(\lambda_{0}-1\right)^{-1}$ and adding this to the right side of (5.2), we find that 


$$
\begin{aligned}
& \left.\frac{d}{d \lambda} s\left(\left(\begin{array}{c}
u^{(0)} \\
p^{(0)}
\end{array}\right),\left(\begin{array}{c}
u^{(0)} \\
p^{(0)}
\end{array}\right) ; \lambda\right)\right|_{\lambda=\lambda_{0}} \\
& =\int_{\Omega}\left(\frac{1+2 \lambda_{0}}{2\left(1-\lambda_{0}\right)^{2}}\left|\nabla_{\omega} u_{r}^{(0)}\right|^{2}-\left(\frac{1+2 \lambda_{0}}{1-\lambda_{0}}+3 \frac{\lambda_{0}+2}{\left(1-\lambda_{0}\right)^{2}}\right)\left|u_{r}^{(0)}\right|^{2}\right. \\
& \left.-\frac{1}{2}\left(2 \lambda_{0}+1\right)\left|u_{\omega}^{(0)}\right|^{2}-\frac{1}{1-\lambda_{0}}\left(u_{r}^{(0)} \nabla_{\omega} \cdot \overline{u_{\omega}^{(0)}}+\left(\nabla_{\omega} \cdot u_{\omega}^{(0)}\right) u_{r}^{(0)}\right)\right) d \omega .
\end{aligned}
$$

We eliminate $\nabla_{\omega} \cdot u_{\omega}^{(0)}$ in the last equation by means of (5.4). Then we obtain

$$
\begin{aligned}
& \left.\frac{d}{d \lambda} s\left(\left(\begin{array}{c}
u^{(0)} \\
p^{(0)}
\end{array}\right),\left(\begin{array}{c}
u^{(0)} \\
p^{(0)}
\end{array}\right) ; \lambda\right)\right|_{\lambda=\lambda_{0}} \\
& =\frac{1+2 \lambda_{0}}{2} \int_{\Omega}\left(\frac{1}{\left(1-\lambda_{0}\right)^{2}}\left|\nabla_{\omega} u_{r}^{(0)}\right|^{2}-\frac{6}{\left(1-\lambda_{0}\right)^{2}}\left|u_{r}^{(0)}\right|^{2}-\left|u_{\omega}^{(0)}\right|^{2}\right) d \omega .
\end{aligned}
$$

Since $s\left(\left(\begin{array}{c}u^{(0)} \\ p^{(0)}\end{array}\right),\left(\begin{array}{c}u^{(0)} \\ p^{(0)}\end{array}\right) ; \lambda_{0}\right)=0$ and $\left(\lambda_{0}+2\right) u_{r}^{(0)}+\nabla_{\omega} \cdot u_{\omega}^{(0)}=0$, it follows that

$$
\begin{aligned}
& -\left.\frac{\left(\lambda_{0}+2\right)\left(1-\lambda_{0}\right)}{1+2 \lambda_{0}} \frac{d}{d \lambda} s\left(\left(\begin{array}{c}
u^{(0)} \\
p^{(0)}
\end{array}\right),\left(\begin{array}{c}
u^{(0)} \\
p^{(0)}
\end{array}\right) ; \lambda\right)\right|_{\lambda=\lambda_{0}} \\
& =s\left(\left(\begin{array}{c}
u^{(0)} \\
p^{(0)}
\end{array}\right),\left(\begin{array}{c}
u^{(0)} \\
p^{(0)}
\end{array}\right) ; \lambda_{0}\right)-\left.\frac{\left(\lambda_{0}+2\right)\left(1-\lambda_{0}\right)}{1+2 \lambda_{0}} \frac{d}{d \lambda} s\left(\left(\begin{array}{c}
u^{(0)} \\
p^{(0)}
\end{array}\right), \quad\left(\begin{array}{c}
u^{(0)} \\
p^{(0)}
\end{array}\right) ; \lambda\right)\right|_{\lambda=\lambda_{0}} \\
& =\left[u_{\omega}, u_{\omega}\right]+\left(\lambda_{0}+2\right)\left(1-\lambda_{0}\right) \int_{\Omega}\left|u_{\omega}^{(0)}\right|^{2} d \omega .
\end{aligned}
$$

The right side of the last equation is nonnegative and equals zero only in the case $u_{\omega}^{(0)}=0, u_{r}^{(0)}=0$. Analogously to the proof of Theorem 3.1 we get $p^{(0)}=0$ in the last case. Hence $\lambda_{0}$ can not be an eigenvalue. This proves the lemma.

THEOREM 5.1. The eigenfunctions of the pencil $\mathfrak{A}(\lambda)$ which correspond to eigenvalues in the strip $|\operatorname{Re} \lambda+1 / 2|<3 / 2$ do not have generalized eigenfunctions.

Proof. By the last assertion of Theorem 3.1 and by Theorem 4.1, it suffices to prove the theorem for real eigenvalues in the interval $(-1 / 2,1)$.

Let $\lambda_{0} \in(-1 / 2,1)$ be an eigenvalue of the pencil $\mathfrak{A}(\lambda),\left(u^{(0)}, p^{(0)}\right)$ an eigenvector to this eigenvalue, and $\left(u^{(1)}, p^{(1)}\right)$ a corresponding generalized eigenvector. Then the equations

$$
a\left(\left(\begin{array}{l}
u^{(0)} \\
p^{(0)}
\end{array}\right),\left(\begin{array}{l}
v \\
q
\end{array}\right) ; \lambda_{0}\right)=0
$$




$$
a\left(\left(\begin{array}{c}
u^{(1)} \\
p^{(1)}
\end{array}\right),\left(\begin{array}{c}
v \\
q
\end{array}\right) ; \lambda_{0}\right)=-\left.\frac{d}{d \lambda} a\left(\left(\begin{array}{c}
u^{(0)} \\
p^{(0)}
\end{array}\right),\left(\begin{array}{l}
v \\
q
\end{array}\right) ; \lambda\right)\right|_{\lambda=\lambda_{0}}
$$

are satisfied for all vector-functions $(v, q) \in \mathscr{H}_{s} \times L_{2}(\Omega)$. Substituting $\left(\begin{array}{c}v \\ q\end{array}\right)=J_{\lambda_{0}}\left(\begin{array}{c}u^{(1)} \\ p^{(1)}\end{array}\right)$ into $(5.6)$, we get

$$
s\left(\left(\begin{array}{c}
u^{(0)} \\
p^{(0)}
\end{array}\right),\left(\begin{array}{c}
u^{(1)} \\
p^{(1)}
\end{array}\right) ; \lambda_{0}\right)=0 .
$$

By the symmetry of the form $s$ we can conclude from this that

$$
0=s\left(\left(\begin{array}{c}
u^{(1)} \\
p^{(1)}
\end{array}\right),\left(\begin{array}{c}
u^{(0)} \\
p^{(0)}
\end{array}\right) ; \lambda_{0}\right)=a\left(\left(\begin{array}{c}
u^{(1)} \\
p^{(1)}
\end{array}\right), J_{\lambda_{0}}\left(\begin{array}{c}
u^{(0)} \\
p^{(0)}
\end{array}\right) ; \lambda_{0}\right) .
$$

Using (5.8) and inserting $\left(\begin{array}{c}v \\ q\end{array}\right)=J_{\lambda_{0}}\left(\begin{array}{c}u^{(0)} \\ p^{(0)}\end{array}\right)$ into (5.7), we obtain

$$
\left.\frac{d}{d \lambda} a\left(\left(\begin{array}{c}
u^{(0)} \\
p^{(0)}
\end{array}\right), J_{\lambda_{0}}\left(\begin{array}{c}
u^{(0)} \\
p^{(0)}
\end{array}\right) ; \lambda\right)\right|_{\lambda=\lambda_{0}}=0 .
$$

From this and (5.6) we get

$$
\left.\frac{d}{d \lambda} a\left(\left(\begin{array}{c}
u^{(0)} \\
p^{(0)}
\end{array}\right), J_{\lambda}\left(\begin{array}{c}
u^{(0)} \\
p^{(0)}
\end{array}\right) ; \lambda\right)\right|_{\lambda=\lambda_{0}}=0 .
$$

Since this contradicts Lemma 5.1, the theorem is proved.

6. The strip $-1 \leq \operatorname{Re} \lambda \leq 0$.

We will show now that the strip $-1 \leq \operatorname{Re} \lambda \leq 0$ may only contain the eigenvalues $\lambda_{0}=0$ and $\lambda_{1}=-1$. Furthermore, we will see that the numbers 0 and -1 are eigenvalues only in some special cases.

THEOREM 6.1. 1) The set

$$
\{\lambda \in \mathrm{C}:-1 \leq \operatorname{Re} \lambda \leq 0\} \backslash\{0,-1\}
$$

does not contain eigenvalues of $\mathfrak{A}(\lambda)$.

2) Let $\mathscr{H}_{c}$ be the set of constant vectors $u \in \mathrm{C}^{3}$ which belong to $\mathscr{H}$. The numbers 0 and -1 are eigenvalues if and only if $\mathscr{H}_{c} \neq\{0\}$. Both values have the same geometric and algebraic multiplicities. The set of the eigenvectors to $\lambda_{0}=0$ coincides with the set $\left\{(u, 0): u \in \mathscr{H}_{c}\right\}$, while generalized eigenvectors do not exist.

Proof. By Theorems 3.1 and 4.1 we can restrict ourselves in the proof to real eigenvalues in the interval $[-1 / 2,0]$.

Suppose that $(u, p)$ is an eigenvector to the eigenvalue $\lambda \in[-1 / 2,0]$. Then according to (3.5) we get 


$$
\begin{aligned}
0 & =s\left(\left(\begin{array}{l}
u \\
p
\end{array}\right),\left(\begin{array}{l}
u \\
p
\end{array}\right) ; \lambda\right) \\
& =\left[u_{\omega}, u_{\omega}\right]+\int_{\Omega} \frac{c}{2}\left(\left|\nabla_{\omega} u_{r}\right|^{2}+\frac{1}{2}(\lambda+2)(1-\lambda)\left|u_{\omega}\right|^{2}-\frac{\lambda+2}{2}\left(\nabla_{\omega} u_{r}\right) \bar{u}_{\omega}\right. \\
& -\frac{\lambda+2}{2} u_{\omega} \cdot \nabla_{\omega} \bar{u}_{r}+(\lambda+2)(1-c \lambda)\left|u_{r}\right|^{2} \\
& \left.+u_{r} \nabla_{\omega} \cdot \bar{u}_{\omega}+\left(\nabla_{\omega} \cdot u_{\omega}\right) \bar{u}_{r}\right) d \omega \\
& =\left[u_{\omega}, u_{\omega}\right]-\frac{1}{2} \int_{\Omega}\left|\nabla_{\omega} \cdot u_{\omega}\right|^{2} d \omega+\int_{\Omega}\left(\frac{\lambda+2}{2(1-\lambda)}\left|\nabla_{\omega} u_{r}-(1-\lambda) u_{\omega}\right|^{2}\right. \\
& \left.+\frac{1}{2}\left|\nabla_{\omega} \cdot u_{\omega}+2 u_{r}\right|^{2}+((\lambda+2)(1-c \lambda)-2)\left|u_{r}\right|^{2}\right) d \omega
\end{aligned}
$$

where as before $c=(\lambda+2) /(1-\lambda)$. By (2.3),

$$
\begin{aligned}
{\left[u_{\omega}, u_{\omega}\right] } & -\frac{1}{2} \int_{\Omega}\left|\nabla_{\omega} \cdot u_{\omega}\right|^{2} d \omega \\
& =\frac{1}{2} \int_{\Omega}\left(\left|\partial_{\theta} u_{\theta}-\frac{1}{\sin \theta} \partial_{\varphi} u_{\varphi}-\cot \theta u_{\theta}\right|^{2}\right. \\
& \left.+\left|\partial_{\theta} u_{\varphi}+\frac{1}{\sin \theta} \partial_{\varphi} u_{\theta}-\cot \theta u_{\varphi}\right|^{2}\right) d \omega \geq 0
\end{aligned}
$$

Since $(\lambda+2) /(1-\lambda)>0$, it follows that $(\lambda+2)(1-c \lambda)-2>0$ for $\lambda \in[-1 / 2,0)$, and (6.1) implies $u_{r}=0, u_{\omega}=0$. This proves the first part of the theorem. If $\lambda=0$, then (6.1) yields

$$
\begin{aligned}
& \nabla_{\omega} \cdot u_{\omega}+2 u_{r}=0, \\
& \nabla_{\omega} u_{r}-u_{\omega}=0, \\
& \partial_{\theta} u_{\theta}-\frac{1}{\sin \theta} \partial_{\varphi} u_{\varphi}-\cot \theta u_{\theta}=0, \\
& \partial_{\theta} u_{\varphi}+\frac{1}{\sin \theta} \partial_{\varphi} u_{\theta}-\cot \theta u_{\varphi}=0
\end{aligned}
$$

Adding the equations (6.2) and (6.4), we get $u_{r}+\partial_{\theta} u_{\theta}=0$. Furthermore, by (6.3) we have $u_{\theta}=\partial_{\theta} u_{r}$ and, consequently, $u_{r}+\partial_{\theta}^{2} u_{r}=0$. Hence the functions $u_{r}, u_{\theta}$ have the form 


$$
\begin{aligned}
& u_{r}=c_{1}(\varphi) \cos \theta+c_{2}(\varphi) \sin \theta, \\
& u_{\theta}=c_{2}(\varphi) \cos \theta-c_{1}(\varphi) \sin \theta .
\end{aligned}
$$

Inserting $u_{\omega}=\nabla_{\omega} u_{r}$ (i.e., $\left.u_{\theta}=\partial_{\theta} u_{r}, u_{\varphi}=(\sin \theta)^{-1} \partial_{\varphi} u_{r}\right)$ into (6.5), we arrive at

$$
\partial_{\theta}\left((\sin \theta)^{-1} \partial_{\varphi} u_{r}\right)=0 .
$$

Consequently, the function $c_{1}$ in the representation of $u_{r}$ is constant. Moreover, it holds $u_{\varphi}=(\sin \theta)^{-1} \partial_{\varphi} u_{r}=c_{2}^{\prime}(\varphi)$. We insert this representation for $u_{\varphi}$ and the representations given above for $u_{r}$ and $u_{\theta}$ into (6.2). Then we obtain

$$
c_{2}(\varphi)+c_{2}^{\prime \prime}(\varphi)=0 \text {. }
$$

This implies $c_{2}=a \cos \varphi+b \sin \varphi$ and

$$
\left(\begin{array}{c}
u_{r} \\
u_{\theta} \\
u_{\varphi}
\end{array}\right)=\left(\begin{array}{ccc}
\sin \theta \cos \varphi & \sin \theta \sin \varphi & \cos \theta \\
\cos \theta \cos \varphi & \cos \theta \sin \varphi & -\sin \theta \\
-\sin \varphi & \cos \varphi & 0
\end{array}\right)\left(\begin{array}{c}
a \\
b \\
c_{1}
\end{array}\right)=J\left(\begin{array}{c}
a \\
b \\
c_{1}
\end{array}\right),
$$

where $a, b, c_{1}$ are constants. Furthermore, for $v_{\omega}=0, q=0$ we have

$$
s\left(\left(\begin{array}{l}
u \\
p
\end{array}\right),\left(\begin{array}{l}
v \\
q
\end{array}\right) ; 0\right)=\int_{\Omega}\left(\nabla_{\omega} u_{r} \cdot \nabla_{\omega} \bar{v}_{r}+\left(2 u_{r}+2 \nabla_{\omega} \cdot u_{\omega}-p\right) \bar{v}_{r}\right) d \omega=0 .
$$

Using (6.2), (6.3) and (3.9), we get $\int_{\Omega} p \bar{v}_{r} d \omega=0$ for all $v_{r} \in \stackrel{\circ}{H}^{1}(\Omega)$, i.e., $p=0$.

Thus, $\lambda_{0}=0$ is the only possible eigenvalue in the strip $-1 / 2 \leq \operatorname{Re} \lambda \leq 0$ and the corresponding eigenvectors (in the Cartesian coordinate system) have the form $(u, 0)$, where $u \in \mathscr{H}_{c}$.

The assertions of the theorem for the number $\lambda_{1}=-1$ follow from Theorem 3.1. The proof is complete.

REMARK 6.1. The condition $\mathscr{H}_{c} \neq\{0\}$ in the second part of Theorem 6.1 is satisfied only in the following three cases:

1. $\mathscr{K}$ is a dihedron bounded by two half-planes $\Gamma_{+}, \Gamma_{-}$and $\mathscr{H}=\left\{u \in H^{1}(\Omega)^{3}: u_{n}=0\right.$ on $\left.\gamma_{ \pm}\right\}$, where $\gamma_{ \pm}=\Gamma_{ \pm} \cap S^{2}$.

2. $\mathscr{K}$ is a dihedron bounded by two half-planes $\Gamma_{+}, \Gamma_{-}$which are orthogonal to each other and $\mathscr{H}=\left\{u \in H^{1}(\Omega)^{3}: u_{n}=0\right.$ on $\gamma_{+}, u_{\tau}=0$ on $\left.\gamma_{-}\right\}$.

3. $\mathscr{K}$ is a half-space bounded by a plane $\Gamma$ and $\mathscr{H}=\left\{u \in H^{1}(\Omega)^{3}, u_{\tau}=0\right.$ on $\left.\gamma=\Gamma \cap S^{2}\right\}$.

In all other cases the numbers 0 and -1 do not belong to the spectrum of the pencil $\mathfrak{A}$. 


\section{The eigenvalue $\lambda=1$.}

In this section we will investigate the number $\lambda=1$. By Theorem 4.1 this is the only possible eigenvalue on the line $\operatorname{Re} \lambda=1$. We will see that the existence and the number of eigenvectors and generalized eigenvectors to $\lambda=1$ depends on the properties of the space $\mathscr{H}$.

Definition 7.1. The space $\mathscr{H} \subset H^{1}(\Omega)^{3}$ is said to be a subspace of the first kind, if

$$
\int_{\partial \Omega} u_{n} d \omega^{\prime}=0 \quad \text { for all } u \in \mathscr{H} .
$$

Otherwise, $\mathscr{H}$ will be called a subspace of the second kind.

REMARK 7.1. Obviously, $\mathscr{H}$ is a subspace of the first kind, if $I_{\tau}=\emptyset$, and a subspace of the second kind, if $I_{\tau} \neq \emptyset$.

We further note that

$$
\int_{\partial \Omega} u_{n} d \omega^{\prime}=\int_{\Omega} \nabla_{\omega} \cdot u_{\omega} d \omega
$$

for arbitrary $u \in H^{1}(\Omega)^{3}$. This equality holds, if we set $v_{r}=1$ in the second part of the proof of Lemma 3.3.

LEMMA 7.1. Let $f$ be an arbitrary function in $L_{2}(\Omega)$.

1) If $\mathscr{H}$ is a subspace of the first kind, then for the solvability of the equation

$$
\nabla_{\omega} \cdot u_{\omega}=f
$$

in space $\mathscr{H}_{s}^{\omega}$ it is necessary and sufficient that

$$
\int_{\Omega} f d \omega=0 .
$$

2) If $\mathscr{H}$ is a subspace of the second kind, then equation (7.1) is always solvable in $\mathscr{H}_{s}^{\omega}$.

3) There exists a constant c such that in both cases a solution $u_{\omega} \in \mathscr{H}_{s}^{\omega}$ of equation (7.1) can be chosen in such a way that

$$
\left\|u_{\omega}\right\|_{h^{1}(\Omega)} \leq c\|f\|_{L_{2}(\Omega)} .
$$

Proof 1) It is evident that the condition (7.2) is necessary for the solvability of (7.1). As it is known (see, e.g. [16]), this condition is also sufficient for the solvability of (7.1) in the space $h^{1}(\Omega)$. This proves the first assertion. 
2) Let $u_{\omega}^{(0)} \in \mathscr{H}_{s}^{\omega}$ and $\int_{\Omega} \nabla_{\omega} \cdot u_{\omega}^{(0)} d \omega \neq 0$. We write the solution $u_{\omega} \in \mathscr{H}_{s}^{\omega}$ of (7.1) in the form

$$
u_{\omega}=u_{\omega}^{(1)}+c u_{\omega}^{(0)} \quad \text { where } c=\left(\int_{\Omega} \nabla_{\omega} \cdot u_{\omega}^{(0)} d \omega\right)^{-1} \int_{\Omega} f d \omega .
$$

Then an equation of the form $\nabla_{\omega} \cdot u_{\omega}^{(1)}=g$ holds for $u_{\omega}^{(1)}$, where $\int_{\Omega} g d \omega=0$. This equation is solvable in $h^{1}(\Omega)$. Thus, the second assertion is proved.

3) We denote by $\mathcal{X}_{0}$ the space $\left\{u_{\omega} \in \mathscr{H}_{s}^{\omega}: \nabla_{\omega} \cdot u_{\omega}=0\right\}$ and by $\mathcal{X}_{1}$ any direct complement in $\mathscr{H}_{s}^{\omega}$. Then by assertions 1), 2) the operator $u_{\omega} \rightarrow \nabla_{\omega} \cdot u_{\omega}$ is an injective mapping from $\mathcal{X}_{1}$ onto the space $\left\{f \in L_{2}(\Omega): \int_{\Omega} f d \omega=0\right\}$, if $\mathscr{H}$ is a subspace of the first kind, and onto $L_{2}(\Omega)$, if $\mathscr{H}$ is a subspace of the second kind. Consequently, if we choose the solution of (7.1) from the space $\mathcal{X}_{1}$, the estimate (7.3) with a constant $c$ independent of $f$ is satisfied. The proof of the lemma is complete.

For $\lambda=1$ the sesquilinear form (3.12) takes the form

$$
\begin{aligned}
a\left(\left(\begin{array}{l}
u \\
p
\end{array}\right),\left(\begin{array}{l}
v \\
q
\end{array}\right) ; 1\right) & =\left[u_{\omega}, v_{\omega}\right]+\int_{\Omega}\left(\frac{1}{2} \nabla_{\omega} u_{r} \cdot \nabla_{\omega} \bar{v}_{r}+\frac{5}{2} u_{r} \nabla_{\omega} \cdot \bar{v}_{\omega}+\left(\nabla_{\omega} \cdot u_{\omega}\right) \bar{v}_{r}\right. \\
& \left.-\frac{1}{2} p \nabla_{\omega} \cdot \bar{v}_{\omega}-\frac{1}{2}\left(3 u_{r}+\nabla_{\omega} \cdot u_{\omega}\right) \bar{q}\right) d \omega .
\end{aligned}
$$

Consequently, every eigenvector $(u, p)$ satisfies the equation

$$
3 u_{r}+\nabla_{\omega} \cdot u_{\omega}=0 \quad \text { in } \Omega
$$

(cf. (3.5)). Inserting $v_{\omega}=0, q=0$ into (7.4) and using (7.5), we obtain

$$
\int_{\Omega}\left(\left(\nabla_{\omega} u_{r}\right) \cdot \nabla_{\omega} \bar{v}_{r}-6 u_{r} \bar{v}_{r}\right) d \omega=0 \quad \text { for all } v_{r} \in \mathscr{H}_{S}^{\omega} .
$$

From (7.4)-(7.6) it follows that

$$
\left[u_{\omega}, v_{\omega}\right]+\int_{\Omega}\left(\frac{5}{2} u_{r} \nabla_{\omega} \cdot \bar{v}_{\omega}-\frac{1}{2} p \nabla_{\omega} \cdot \bar{v}_{\omega}\right) d \omega=0 \quad \text { for all } v_{\omega} \in \mathscr{H}_{s}^{\omega} .
$$

The equation (7.7) together with (7.5) can be considered as a variational problem for the vector-function $\left(u_{\omega}, p\right)$. We consider the following generalization of this problem.

Problem 1. Let the functions $f, g \in L_{2}(\Omega)$ and the vector-function $F \in L_{2}(\Omega)^{2}$ be given. One has to find a vector-function $\left(u_{\omega}, p\right) \in \mathscr{H}_{s}^{\omega} \times L_{2}(\Omega)$ satisfying the integral identity 


$$
\begin{aligned}
& {\left[u_{\omega}, v_{\omega}\right]+\int_{\Omega}\left(-\frac{1}{2} p \nabla_{\omega} \cdot \bar{v}_{\omega}+\left(\nabla_{\omega} \cdot u_{\omega}\right) \bar{q}\right) d \omega} \\
& =\int_{\Omega}\left(f \nabla_{\omega} \cdot \bar{v}_{\omega}+F \cdot \bar{v}_{\omega}+g \bar{q}\right) d \omega
\end{aligned}
$$

for all $v_{\omega} \in \mathscr{H}_{s}^{\omega}, q \in L_{2}(\Omega)$.

REMARK 7.2. From the integral identity (7.8) it follows that

$$
\nabla_{\omega} \cdot u_{\omega}=g \quad \text { in } \Omega .
$$

Therefore, (7.8) can be written in the form

$$
\begin{aligned}
& {\left[u_{\omega}, v_{\omega}\right]-\frac{1}{2} \int_{\Omega} p \nabla_{\omega} \cdot \bar{v}_{\omega} d \omega} \\
& =\int_{\Omega}\left(f \nabla_{\omega} \cdot \bar{v}_{\omega}+F \cdot \bar{v}_{\omega}\right) d \omega \text { for all } v_{\omega} \in \mathscr{H}_{s}^{\omega} .
\end{aligned}
$$

Clearly, the integral identity (7.8) is equivalent to the validity of the equality (7.9) and the relation (7.10).

Before we investigate the solvability of Problem 1, we prove the following lemma.

LEMmA 7.2. The set of the vector-functions $u_{\omega} \in h^{1}(\Omega)$ satisfying the equation

$$
\left[u_{\omega}, u_{\omega}\right]=0
$$

is exhausted by the linear combinations of the vector-functions

$$
u_{\omega}^{(1)}=\left(\begin{array}{c}
\sin \varphi \\
\cos \theta \cos \varphi
\end{array}\right), \quad u_{\omega}^{(2)}=\left(\begin{array}{c}
\cos \varphi \\
-\cos \theta \sin \varphi
\end{array}\right), \quad u_{\omega}^{(3)}=\left(\begin{array}{c}
0 \\
\sin \theta
\end{array}\right) .
$$

Proof. Equation (7.11) is equivalent to

$$
\begin{aligned}
& \partial_{\theta} u_{\theta}=0 \quad \text { in } \Omega, \\
& \partial_{\varphi} u_{\varphi}+\cos \theta u_{\theta}=0 \quad \text { in } \Omega, \\
& \partial_{\theta}\left(\frac{u_{\varphi}}{\sin \theta}\right)+\frac{1}{\sin ^{2} \theta} \partial_{\varphi} u_{\theta}=0 \quad \text { in } \Omega .
\end{aligned}
$$

From (7.13) it follows that $u_{\theta}=u_{\theta}(\varphi)$. Hence from (7.14) we conclude that $u_{\varphi}$ has the form

$$
u_{\varphi}=-\cos \theta F(\varphi)+h(\theta), \quad \text { where } F^{\prime}=u_{\theta} .
$$


Using now (7.15), we find that

$$
u_{\varphi}=\cos \theta \partial_{\varphi} u_{\theta}+\sin \theta g(\varphi) .
$$

Consequently,

$$
\cos \theta\left(\partial_{\varphi} u_{\theta}+F(\varphi)\right)+\sin \theta g(\varphi)=h(\theta)
$$

This implies

$$
F=a \cos \varphi+b \sin \varphi+c_{1}, \quad g=c_{2}, \quad h(\theta)=c_{1} \cos \theta+c_{2} \sin \theta,
$$

where $a, b, c_{1}, c_{2}$ are constants. Thus, we get

$$
u_{\theta}(\varphi)=b \cos \varphi-a \sin \varphi, \quad u_{\varphi}=-\cos \theta(a \cos \varphi+b \sin \varphi)+c_{2} \sin \theta .
$$

This proves the lemma.

REMARK 7.3. 1) By the inequality (2.3), we have $\nabla_{\omega} \cdot u_{\omega}=0$ for every vector-function $u_{\omega} \in h^{1}(\Omega)$ satisfying (7.11).

2) Let $u_{\omega}^{(j)}, j=1,2,3$, be the vectors (7.12). Then the vectors $r\left(\begin{array}{c}0 \\ u_{\omega}^{(j)}\end{array}\right)$, $j=1,2,3$, have the form of rigid body rotations

$$
\left(\begin{array}{c}
0 \\
x_{3} \\
-x_{2}
\end{array}\right), \quad\left(\begin{array}{c}
x_{3} \\
0 \\
-x_{1}
\end{array}\right), \quad\left(\begin{array}{c}
-x_{2} \\
x_{1} \\
0
\end{array}\right)
$$

in the Cartesian system of coordinates.

We denote by $\mathscr{H}_{0}$ the set of all linear combinations of the vector-functions (7.12) which belong to the space $\mathscr{H}_{s}^{\omega}$.

Lemma 7.3. Let $f=g=0$ and $F=0$. Then the following assertions are valid for the solutions of Problem 1.

1) If $\mathscr{H}$ is a subspace of the first kind, then the solutions of Problem 1 are exhausted by the vector-functions $\left(u_{\omega}, p\right)$, where $u_{\omega} \in \mathscr{H}_{0}, p=$ const $\in \mathrm{C}$.

2) If $\mathscr{H}$ is a subspace of the second kind, then the solutions of Problem 1 are exhausted by the vector-functions $\left(u_{\omega}, 0\right)$, where $u_{\omega} \in \mathscr{H}_{0}$.

Proof For $f=g=0, F=0$ from (7.8) it follows that $\nabla_{\omega} \cdot u_{\omega}=0$ and

$$
\left[u_{\omega}, v_{\omega}\right]-\frac{1}{2} \int_{\Omega} p \nabla_{\omega} \cdot \bar{v}_{\omega} d \omega=0 \quad \text { for all } v \in \mathscr{H}_{s}^{\omega}
$$

(see Remark 7.2). Consequently, $\left[u_{\omega}, u_{\omega}\right]=0$. This implies $\left[u_{\omega}, v_{\omega}\right]=0$ for all $v_{\omega} \in \mathscr{H}_{s}^{\omega}$ and 


$$
\int_{\Omega} p \nabla_{\omega} \cdot \bar{v}_{\omega} d \omega=0 \quad \text { for all } v_{\omega} \in \mathscr{H}_{s}^{\omega}
$$

Since $\stackrel{\circ}{h}^{1}(\Omega) \subset \mathscr{H}_{s}^{\omega}$, the last equality can be satisfied only for constant $p$. Now the assertions of the lemma follow from Definition 7.1 and Lemma 7.2.

We consider the inhomogeneous Problem 1 now.

LeMma 7.4 Let $f, g \in L_{2}(\Omega)$ and $F \in L_{2}(\Omega)^{2}$.

1) If $\mathscr{H}$ is a subspace of the first kind, then for the solvability of Problem 1 it is necessary and sufficient that

$$
\int_{\Omega} g d \omega=0 \quad \text { and } \quad \int_{\Omega} F \cdot \bar{v}_{\omega} d \omega=0 \text { for all } v_{\omega} \in \mathscr{H}_{0} .
$$

2) If $\mathscr{H}$ is a subspace of the second kind, then the condition

$$
\int_{\Omega} F \cdot \bar{v}_{\omega} d \omega=0 \text { for all } v_{\omega} \in \mathscr{H}_{0} .
$$

is necessary and sufficient for the solvability of Problem 1.

Proof. The proof of the necessity of the conditions (7.16), (7.17) is obvious. We prove the sufficiency of these conditions.

Let at first $\mathscr{H}$ be a subspace of the first kind. As we have mentioned in Remark 7.2, the solution $\left(u_{\omega}, p\right)$ of Problem 1 satisfies the equation $\nabla_{\omega} \cdot u_{\omega}=g$. Using the first condition in (7.16) and the first part of Lemma 7.1 , we obtain the existence of a solution $u_{\omega}^{(0)}$ of the equation $\nabla_{\omega} \cdot u_{\omega}=g$ in the space $\mathscr{H}_{s}^{\omega}$. We write the solution of Problem 1 in the form $u_{\omega}=u_{\omega}^{(1)}+u_{\omega}^{(0)}$. Then for the determination of $u_{\omega}^{(1)}$ and $p$ we have the equation

$$
\begin{aligned}
{\left[u_{\omega}^{(1)}, v_{\omega}\right] } & -\frac{1}{2} \int_{\Omega} p \nabla_{\omega} \cdot \bar{v}_{\omega} d \omega=-\left[u_{\omega}^{(0)}, v_{\omega}\right] \\
& +\int_{\Omega}\left(f \nabla_{\omega} \cdot \bar{v}_{\omega}+F \cdot \bar{v}_{\omega}\right) d \omega \text { for all } v_{\omega} \in \mathscr{H}_{s}^{\omega} .
\end{aligned}
$$

Furthermore, the equation $\nabla_{\omega} \cdot u_{\omega}^{(1)}=0$ must be satisfied in $\Omega$.

We introduce the space $\mathcal{X}=\left\{u_{\omega} \in \mathscr{H}_{s}^{\omega}: \nabla_{\omega} \cdot u_{\omega}=0\right\}$. It is evident that $\mathscr{H}_{0} \subset \mathcal{X}$. Let $\mathcal{X}_{0}$ be the direct complement of $\mathscr{H}_{0}$ in $\mathcal{X}$. Then $[\cdot, \cdot]$ is a scalar product in $\mathcal{X}_{0}$. We seek the element $u_{\omega}^{(1)}$ in the space $\mathcal{X}_{0}$. Inserting $v_{\omega} \in \mathcal{X}_{0}$ into (7.18), we obtain 


$$
\left[u_{\omega}^{(1)}, v_{\omega}\right]=-\left[u_{\omega}^{(0)}, v_{\omega}\right]+\int_{\Omega} F \cdot \bar{v}_{\omega} d \omega \quad \text { for all } v_{\omega} \in \mathcal{X}_{0} .
$$

The right side of (7.19) is a bounded antilinear functional in $\mathcal{X}_{0}$. Hence the Riesz theorem implies the existence of an element $u_{\omega}^{(1)} \in \mathcal{X}_{0}$ satisfying (7.19).

If $v_{\omega} \in \mathscr{H}_{0}$, then (7.19) is satisfied by the second condition of (7.16). Consequently, (7.19) is satisfied for all $v_{\omega} \in \mathcal{X}$.

Let $\mathcal{X}_{1}$ be the direct complement of the subspace $\mathcal{X}$ in $\mathscr{H}_{s}^{\omega}$ and let $\mathscr{Y}=\left\{g \in L_{2}(\Omega): \int_{\Omega} g d \omega=0\right\}$. By the first part of Lemma 7.1 the operator $u_{\omega} \rightarrow \nabla_{\omega} \cdot u_{\omega}$ is an isomorphism from $\mathcal{X}_{1}$ onto $\mathscr{Y}$. We represent the element $v_{\omega} \in \mathscr{H}_{s}^{\omega}$ as a sum $v_{\omega}=v_{\omega}^{(0)}+v_{\omega}^{(1)}$, where $v_{\omega}^{(0)} \in \mathcal{X}, v_{\omega}^{(1)} \in \mathcal{X}_{1}$. Then the integral identity (7.18) is satisfied, if

$$
\begin{aligned}
-\frac{1}{2} \int_{\Omega} p \nabla_{\omega} \cdot \overline{v_{\omega}^{(1)}} d \omega & =-\left[u_{\omega}^{(0)}, v_{\omega}^{(1)}\right]+\int_{\Omega} F \cdot \overline{v_{\omega}^{(1)}} d \omega-\left[u_{\omega}^{(1)}, v_{\omega}^{(1)}\right] \\
& +\int_{\Omega} f \nabla_{\omega} \cdot \overline{v_{\omega}^{(1)}} d \omega .
\end{aligned}
$$

The right side of (7.20) is a functional on $\mathscr{X}_{1}$ and, consequently, also on $\mathscr{Y}$. Therefore, there exists an element $p \in \mathscr{Y}$ satisfying (7.20) for all $v_{\omega}^{(1)} \in \mathscr{X}_{1}$. Thus, we have proved part 1) of the lemma.

The proof of the second part proceeds analogously.

Now we are able to give a description of the eigenfunctions and generalized eigenfunctions corresponding to the eigenvalue $\lambda=1$. We start with the case when $\mathscr{H}$ is a subspace of the first kind.

Theorem 7.1. Suppose that $\Omega \neq S^{2}$ and $\mathscr{H}$ is a subspace of the first kind. Then the number $\lambda=1$ is always an eigenvalue of the pencil $\mathfrak{A}(\lambda)$ and the vector-functions $\left(0, u_{\omega}, c\right)$, where $u_{\omega} \in \mathscr{H}_{0}, c \in \mathrm{C}$, are corresponding eigenvectors. Moreover, the following assertions are true.

1) If the problem

$$
\left\{\begin{array}{l}
\int_{\Omega}\left(\nabla_{\omega} w \cdot \nabla_{\omega} \bar{v}-6 w \bar{v}\right) d \omega=0 \text { for all } v \in \mathscr{H}_{s}^{\omega}, \\
\int_{\Omega} w d \omega=0
\end{array}\right.
$$

has only the trivial solution in the space $\mathscr{H}_{s}^{r}$, then there are no other eigenvectors. If the problem (7.21) has a nontrivial solution $w \in \mathscr{H}_{s}^{r}$, then there are the additional eigenvectors $\left(w, u_{\omega}, p\right)$, where $\left(u_{\omega}, p\right)$ is a solution of Problem 1 with 
$f=-\frac{5}{2} w, g=-3 w, F=0$. Other eigenvectors to the eigenvalue $\lambda=1$ do not occur.

2) For the existence of a generalized eigenvector to the eigenvector $\left(u_{r}^{(0)}, u_{\omega}^{(0)}, p^{(0)}\right)$ it is necessary and sufficient that $u_{r}^{(0)}=0, u_{\omega}^{(0)}=0, p^{(0)}=$ const, and that the problem

$$
\left\{\begin{array}{l}
\int_{\Omega}\left(\nabla_{\omega} w \cdot \nabla_{\omega} \bar{v}-6 w \bar{v}\right) d \omega=-\int_{\Omega} p^{(0)} \bar{v} d \omega \text { for all } v \in \mathscr{H}_{s}^{r} \\
\int_{\Omega} w d \omega=0
\end{array}\right.
$$

has a solution $w \in \mathscr{H}_{s}^{r}$. In this case the corresponding generalized eigenvector has the form $\left(w, u_{\omega}, p\right)$, where $\left(u_{\omega}, p\right)$ is a solution of Problem 1 with $f=-\frac{5}{2} w$, $g=-3 w, F=0$. Other generalized eigenvectors do not occur.

Proof. Let $\left(u_{r}, u_{\omega}, p\right)$ be an eigenvector to the eigenvalue $\lambda=1$. If $u_{r}=0$, then by (7.5) we get $\nabla_{\omega} \cdot u_{\omega}=0$ and from (7.7) it follows that

$$
\left[u_{\omega}, u_{\omega}\right]-\frac{1}{2} \int_{\Omega} p \nabla_{\omega} \cdot v_{\omega} d \omega=0 \text { for all } v_{\omega} \in \mathscr{H}_{s}^{\omega} .
$$

Using the first part of Lemma 7.3, we conclude that $u_{\omega} \in \mathscr{H}_{0}$ and $p=$ const.

Let now $u_{r}$ be nonzero. Then by (7.5) and (7.6) the function $u_{r}$ is a solution of the problem (7.21) and from (7.5), (7.7) it follows that the vector-function $\left(u_{\omega}, p\right)$ is a solution of the problem

$$
\begin{aligned}
& \nabla_{\omega} \cdot u_{\omega}=-3 u_{r} \\
& {\left[u_{\omega}, v_{\omega}\right]-\frac{1}{2} \int_{\Omega} p \nabla_{\omega} \cdot \bar{v}_{\omega} d \omega=-\frac{5}{2} \int_{\Omega} u_{r} \nabla_{\omega} \cdot \bar{v}_{\omega} d \omega \text { for all } v_{\omega} \in \mathscr{H}_{s}^{\omega} .}
\end{aligned}
$$

This proves the assertions on the eigenvectors.

We investigate now the existence of generalized eigenvectors. Let $\left(u_{r}^{(0)}, u_{\omega}^{(0)}, p^{(0)}\right)$ be an eigenvector to the eigenvalue $\lambda=1$ and let $\left(u_{r}^{(1)}, u_{\omega}^{(1)}, p^{(1)}\right)$ be a corresponding generalized eigenvector. Differentiating (3.12) with respect to $\lambda$, we obtain

$$
\begin{aligned}
\left.\frac{d}{d \lambda} a\left(\left(\begin{array}{c}
u \\
p
\end{array}\right),\left(\begin{array}{c}
v \\
q
\end{array}\right) ; \lambda\right)\right|_{\lambda=1} & =\frac{1}{2} \int_{\Omega}\left(-6 u_{r} \bar{v}_{r}-3 u_{\omega} \cdot \bar{v}_{\omega}+u_{r} \nabla_{\omega} \cdot \bar{v}_{\omega}\right. \\
& \left.-\left(\nabla_{\omega} \cdot u_{\omega}\right) \bar{v}_{r}+p \bar{v}_{r}-u_{r} \bar{q}\right) d \omega .
\end{aligned}
$$

Consequently, the generalized eigenfunction satisfies the equation 


$$
\begin{aligned}
{\left[u_{\omega}^{(1)}, v_{\omega}\right] } & +\frac{1}{2} \int_{\Omega}\left(\left(\nabla_{\omega} u_{r}^{(1)}\right) \cdot \nabla_{\omega} \bar{v}_{r}+5 u_{r}^{(1)} \nabla_{\omega} \cdot \bar{v}_{\omega}\right. \\
& +2\left(\nabla_{\omega} \cdot u_{\omega}^{(1)}\right) \bar{v}_{r}-p^{(1)} \nabla_{\omega} \cdot \bar{v}_{\omega} \\
& \left.-\left(3 u_{r}^{(1)}+\nabla_{\omega} \cdot u_{\omega}^{(1)}\right) \bar{q}\right) d \omega \\
& +\frac{1}{2} \int_{\Omega}\left(-6 u_{r}^{(0)} \bar{v}_{r}-3 u_{\omega}^{(0)} \cdot \bar{v}_{\omega}+u_{r}^{(0)} \nabla_{\omega} \cdot \bar{v}_{\omega}\right. \\
& \left.-\left(\nabla_{\omega} \cdot u_{\omega}^{(0)}\right) \bar{v}_{r}+p^{(0)} \bar{v}_{r}-u_{r}^{(0)} \bar{q}\right) d \omega=0
\end{aligned}
$$

for all $v_{r} \in \mathscr{H}_{s}^{r}, v_{\omega} \in \mathscr{H}_{s}^{\omega}, q \in L_{2}(\Omega)$. From this we get

$$
3 u_{r}^{(1)}+\nabla_{\omega} \cdot u_{\omega}^{(1)}+u_{r}^{(0)}=0 \quad \text { in } \Omega
$$

and

$$
\begin{array}{r}
\int_{\Omega}\left(\left(\nabla_{\omega} u_{r}^{(1)}\right) \cdot \nabla_{\omega} \bar{v}_{r}+2\left(\nabla_{\omega} \cdot u_{\omega}^{(1)}\right) \bar{v}_{r}-6 u_{r}^{(0)} \bar{v}_{r}\right. \\
\left.-\left(\nabla_{\omega} \cdot u_{\omega}^{(0)}\right) \bar{v}_{r}+p^{(0)} \bar{v}_{r}\right) d \omega=0
\end{array}
$$

for all $\quad v_{r} \in \mathscr{H}_{s}^{r}$. Using the equalities $\nabla_{\omega} \cdot u_{\omega}^{(0)}=-3 u_{r}^{(0)} \quad$ and $\nabla_{\omega} \cdot u_{\omega}^{(1)}=-3 u_{r}^{(1)}-u_{r}^{(0)}$ (see (7.5), (7.25)), we find that (7.26) is equivalent to

$$
\int_{\Omega}\left(\left(\nabla_{\omega} u_{r}^{(1)}\right) \cdot \nabla_{\omega} \bar{v}_{r}-6 u_{r}^{(1)} \bar{v}_{r}\right) d \omega=\int_{\Omega}\left(5 u_{r}^{(0)} \bar{v}_{r}-p^{(0)} \bar{v}_{r}\right) d \omega
$$

for $v_{r} \in \mathscr{H}_{s}^{r}$. Substituting $v_{r}=u_{r}^{(0)}$ into (7.27) and using (7.6), we obtain

$$
\int_{\Omega}\left(5\left|u_{r}^{(0)}\right|^{2}-p^{(0)} \overline{u_{r}^{(0)}}\right) d \omega=0 .
$$

Furthermore, by (7.7) we have

$$
\left[u_{\omega}^{(0)}, u_{\omega}^{(0)}\right]+\frac{1}{2} \int_{\Omega}\left(5 u_{r}^{(0)} \nabla_{\omega} \cdot \overline{u_{\omega}^{(0)}}-p^{(0)} \nabla_{\omega} \cdot \overline{u_{\omega}^{(0)}}\right) d \omega=0 .
$$

The last equality together with the equality $\nabla_{\omega} \cdot u_{\omega}^{(0)}=-3 u_{r}^{(0)}$ and (7.28) implies $\left[u_{\omega}^{(0)}, u_{\omega}^{(0)}\right]=0$. Therefore, $u_{\omega}^{(0)} \in \mathscr{H}_{0}$. Moreover, we get $u_{r}^{(0)}=-\frac{1}{3} \nabla_{\omega} \cdot u_{\omega}^{(0)}=0$ (see Remark 7.3). As it has been shown in the beginning of the proof, this implies $p^{(0)}=$ const. Inserting $v_{r}=0, q=0$ into (7.24), we find 


$$
\left[u_{\omega}^{(1)}, v_{\omega}\right]+\frac{1}{2} \int_{\Omega}\left(5 u_{r}^{(1)} \nabla_{\omega} \cdot \bar{v}_{\omega}-p^{(1)} \nabla_{\omega} \cdot \bar{v}_{\omega}-3 u_{\omega}^{(0)} \cdot \bar{v}_{\omega}\right) d \omega=0
$$

for all $v_{\omega} \in \mathscr{H}_{s}^{\omega}$.

In particular, if $v_{\omega} \in \mathscr{H}_{0}$, then

$$
\int_{\Omega} u_{\omega}^{(0)} \cdot \bar{v}_{\omega} d \omega=0
$$

and, therefore, $u_{\omega}^{(0)}=0$. Thus, we have shown that only eigenvectors of the form $\left(0,0, p^{(0)}\right)$ may have generalized eigenvectors. In this case the following relations for the generalized eigenvectors hold (see (7.25), (7.27), (7.29)):

$$
\begin{aligned}
& 3 u_{r}^{(1)}+\nabla_{\omega} \cdot u_{\omega}^{(1)}=0 \quad \text { in } \Omega \\
& \int_{\Omega}\left(\left(\nabla_{\omega} u_{r}^{(1)}\right) \cdot \nabla_{\omega} \bar{v}_{r}-6 u_{r}^{(1)} \bar{v}_{r}+p^{(0)} \bar{v}_{r}\right) d \omega=0 \quad \text { for all } v_{r} \in \mathscr{H}_{s}^{r}, \\
& {\left[u_{\omega}^{(1)}, v_{\omega}\right]+\frac{1}{2} \int_{\Omega}\left(5 u_{r}^{(1)} \nabla_{\omega} \cdot \bar{v}_{\omega}-p^{(1)} \nabla_{\omega} \cdot \bar{v}_{\omega}\right) d \omega=0 \text { for all } v_{\omega} \in \mathscr{H}_{s}^{\omega} .}
\end{aligned}
$$

From (7.30), (7.31) it follows that $u_{r}^{(1)}$ is a solution of the problem (7.22), while (7.32) is equivalent to Problem 1 for $f=-\frac{5}{2} u_{r}^{(1)}, g=-3 u_{r}^{(1)}, F=0$ due to $(7.30)$.

We will now show that there are no second generalized eigenvectors. Let $\left(u_{r}^{(1)}, u_{\omega}^{(1)}, p^{(1)}\right)$ be a generalized eigenvector to the eigenvector $\left(0,0, p^{(0)}\right)$, $p^{(0)}=$ const. Suppose there exists a second generalized eigenvector $\left(u_{r}^{(2)}, u_{\omega}^{(2)}, p^{(2)}\right)$. Since

$$
\left.\frac{d^{2}}{d \lambda^{2}} a\left(\left(\begin{array}{l}
u \\
p
\end{array}\right),\left(\begin{array}{l}
v \\
q
\end{array}\right) ; \lambda\right)\right|_{\lambda=1}=\int_{\Omega}\left(-2 u_{r} \bar{v}_{r}-u_{\omega} \cdot \bar{v}_{\omega}\right) d \omega,
$$

it follows that

$$
\begin{aligned}
{\left[u_{\omega}^{(2)}, v_{\omega}\right] } & +\frac{1}{2} \int_{\Omega}\left(\left(\nabla_{\omega} u_{r}^{(2)}\right) \cdot \nabla_{\omega} \bar{v}_{r}+5 u_{r}^{(2)} \nabla_{\omega} \cdot \bar{v}_{\omega}+2\left(\nabla_{\omega} \cdot u_{\omega}^{(2)}\right) \bar{v}_{r}-p^{(2)} \nabla_{\omega} \cdot \bar{v}_{\omega}\right. \\
& -\left(3 u_{r}^{(2)}+\nabla_{\omega} \cdot u_{\omega}^{(2)}\right) \bar{q}-6 u_{r}^{(1)} \bar{v}_{r}-3 u_{\omega}^{(1)} \cdot \bar{v}_{\omega}+u_{r}^{(1)} \nabla_{\omega} \cdot \bar{v}_{\omega} \\
& \left.-\left(\nabla_{\omega} \cdot u_{\omega}^{(1)}\right) \bar{v}_{r}+p^{(1)} \bar{v}_{r}-u_{r}^{(1)} \bar{q}\right) d \omega=0
\end{aligned}
$$

for all $v_{r} \in \mathscr{H}_{s}^{r}, v_{\omega} \in \mathscr{H}_{s}^{\omega}, q \in L_{2}(\Omega)$. This implies

$$
3 u_{r}^{(2)}+\nabla_{\omega} \cdot u_{\omega}^{(2)}+u_{r}^{(1)}=0 \quad \text { in } \Omega
$$


and

$$
\begin{aligned}
& \int_{\Omega}\left(\left(\nabla_{\omega} u_{r}^{(2)}\right) \cdot \nabla_{\omega} \bar{v}_{r}-6 u_{r}^{(2)} \bar{v}_{r}\right) d \omega \\
& =\int_{\Omega}\left(5 u_{r}^{(1)} \bar{v}_{r}-p^{(1)} \bar{v}_{r}\right) d \omega \text { for all } v_{r} \in \mathscr{H}_{s}^{r} .
\end{aligned}
$$

From (7.25), (7.33) and from the properties of the space $\mathscr{H}_{s}^{\omega}$ it follows that $\int_{\Omega} u_{r}^{(2)} d \omega=0$. Hence by (7.27) we have

$$
\int_{\Omega}\left(\nabla_{\omega} u_{r}^{(2)} \cdot \nabla_{\omega} \overline{u_{r}^{(1)}}-6 u_{r}^{(2)} \overline{u_{r}^{(1)}}\right) d \omega=-\overline{p^{(0)}} \int_{\Omega} u_{r}^{(2)} d \omega=0 .
$$

Inserting $v_{r}=u_{r}^{(1)}$ into (7.34), we, consequently, obtain

$$
\int_{\Omega}\left(5\left|u_{r}^{(1)}\right|^{2}-p^{(1)} \overline{u_{r}^{(1)}}\right) d \omega=0 .
$$

We now insert $v_{\omega}=u_{\omega}^{(1)}$ into (7.32). Then (7.30) yields

$$
\left[u_{\omega}^{(1)}, u_{\omega}^{(1)}\right]-\frac{3}{2} \int_{\Omega}\left(5\left|u_{r}^{(1)}\right|^{2}-p^{(1)} \overline{u_{r}^{(1)}}\right) d \omega=0 .
$$

From this and (7.35) we conclude that $\left[u_{\omega}^{(1)}, u_{\omega}^{(1)}\right]=0$, i.e., $u_{\omega}^{(1)} \in \mathscr{H}_{0}$. According to (7.30) and Remark 7.3 this implies $u_{r}^{(1)}=0$. Thus, second generalized eigenvectors do not exist. The proof is complete.

THEOREM 7.2. Let $\Omega \neq S^{2}$ and let $\mathscr{H}$ be a subspace of the second kind.

1) If $\mathscr{H}_{0} \neq\{0\}$, then the number $\lambda=1$ is always an eigenvalue of the pencil $\mathfrak{U}(\lambda)$. The vectors $\left(0, u_{\omega}, 0\right)$, where $u_{\omega} \in \mathscr{H}_{0}$, are eigenvectors to this eigenvalue. If the problem

$$
\int_{\Omega}\left(\nabla_{\omega} w \cdot \nabla_{\omega} \bar{v}-6 w \bar{v}\right) d \omega=0 \quad \text { for all } v \in \mathscr{H}_{s}^{r}
$$

has only the trivial solution $w=0$ in $\mathscr{H}_{s}^{r}$, then there are no other eigenvectors. If the problem (7.36) has a nontrivial solution $w \in \mathscr{H}_{s}^{r}$, then there is also the eigenvector $\left(w, u_{\omega}, p\right)$, where $\left(u_{\omega}, p\right)$ is a solution of Problem 1 with $f=-\frac{5}{2} w$, $g=-3 w, F=0$. Generalised eigenvectors do not occur.

2) If $\mathscr{H}_{0}=\{0\}$, then $\lambda=1$ is an eigenvalue if and only if the problem (7.36) has a nontrivial solution $w \in \mathscr{H}_{s}^{r}$. Then there is the eigenvector $\left(w, u_{\omega}, p\right)$, where 
$\left(u_{\omega}, p\right)$ is a solution of Problem 1 with $f=-\frac{5}{2} w, g=-3 w, F=0$. Generalised eigenvectors do not occur.

Proof. First we investigate the existence and the form of the eigenvectors. Let $\left(u_{r}, u_{\omega}, p\right)$ be an eigenvector of the pencil $\mathfrak{A}(\lambda)$ to the eigenvalue $\lambda=1$. Then analogously to the proof of Theorem 7.1, the functions $u_{r}, u_{\omega}, p$ satisfy (7.23). If $u_{r}=0$, then by the second part of Lemma 7.3, we get $u_{\omega} \in \mathscr{H}_{0}$ and $p=0$. Consequently, eigenvectors of the form $\left(0, u_{\omega}, p\right)$ exist if and only if $\mathscr{H}_{0} \neq\{0\}$. Suppose that $u_{r} \neq 0$. Then by (7.6) the function $u_{r}$ is a nontrivial solution of the problem (7.36), while $\left(u_{\omega}, p\right)$ is a solution of the problem (7.23). This proves the assertions of the theorem concerning the eigenvectors to the eigenvalue $\lambda=1$.

Now we show that generalized eigenvectors do not exist. For this end, we assume that $\left(u_{r}^{(0)}, u_{\omega}^{(0)}, p^{(0)}\right)$ is an eigenvector to the eigenvalue $\lambda=1$ and that $\left(u_{r}^{(1)}, u_{\omega}^{(1)}, p^{(1)}\right)$ is a corresponding generalized eigenvector. Analogously to the proof of Theorem 7.1 we find that $u_{\omega}^{(0)}=0$ and, consequently, $\nabla_{\omega} \cdot u_{\omega}^{(0)}=0$. Hence by (5.4) we have $u_{r}^{(0)}=0$. As we have shown in the beginning of the proof, this implies $p^{(0)}=0$. Furthermore, analogously to the proof of Theorem 7.1 the equality (7.29) is satisfied. In particular, we have

$$
\int_{\Omega} u_{\omega}^{(0)} \cdot \bar{v}_{\omega} d \omega=0 \quad \text { for all } v_{\omega} \in \mathscr{H}_{0}
$$

and, therefore, $u_{\omega}^{(0)}=0$. Thus, the vector $\left(u_{r}^{(0)}, u_{\omega}^{(0)}, p^{(0)}\right)$ is not an eigenvector. This proves the theorem.

\section{A variational principle for real eigenvalues.}

In this section we get a variational principle for real eigenvalues of the operator pencil $\mathfrak{H}(\lambda)$ lying in the interval $-1 / 2 \leq \lambda<1$. The derivation of this variational principle is analogous to [11]. For this reason, we give the formulation of the following lemmas and theorems without proof.

We introduce the space

$$
\mathscr{H}_{s}^{(\lambda)}=\left\{u \in \mathscr{H}_{s}:(\lambda+2) u_{r}+\nabla_{\omega} \cdot u_{\omega}=0\right\}
$$

for any fixed $\lambda \in[-1 / 2,1)$. For vector-functions $u, v \in \mathscr{H}_{s}^{(\lambda)}$ the sesquilinear form (4.1) is independent of $p$ and $q$ and takes the form 


$$
\begin{aligned}
t(u, v ; \lambda) & :=\left[u_{\omega}, v_{\omega}\right]+\int_{\Omega}\left(\frac{c}{2}\left(\nabla_{\omega} u_{r}\right) \cdot \nabla_{\omega} \bar{v}_{r}+(\lambda+2)(1-c \lambda) u_{r} \bar{v}_{r}\right. \\
& +\frac{1}{2}(\lambda+2)(1-\lambda) u_{\omega} \cdot \bar{v}_{\omega} \\
& \left.+\left(\frac{\lambda+2}{2}+1\right)\left(u_{r} \nabla_{\omega} \cdot \bar{v}_{\omega}+\left(\nabla_{\omega} \cdot u_{\omega}\right) \bar{v}_{r}\right)\right) d \omega .
\end{aligned}
$$

The form $t(\cdot, \cdot ; \lambda)$ induces a self-adjoint operator $T_{\lambda}$ on $\mathscr{H}_{s}{ }^{(\lambda)}$ which is bounded from below and has discrete spectrum. We denote by $m_{1}(\lambda) \leq m_{2}(\lambda) \leq \cdots$ the eigenvalues of $T_{\lambda}$ counted according to their multiplicities. These eigenvalue are given by the equality

$$
m_{j}(\lambda)=\max _{L} \min _{u \in L \backslash\{0\}} \frac{t(u, u ; \lambda)}{\int_{\Omega}|u|^{2} d \omega},
$$

where the maximum is taken over all subspaces $L \subset \mathscr{H}_{s}^{(\lambda)}$ of codimension $\geq j-1$.

Since the form $t(\cdot, \cdot ;-1 / 2)$ is positive, we have $m_{j}(-1 / 2)>0$.

Let $\mathscr{Y}$ be the space $\left\{u_{\omega} \in \mathscr{H}_{s}{ }^{\omega}: \nabla_{\omega} \cdot u_{\omega} \in \mathscr{H}_{s}^{r}\right\}$. Eliminating the function $u_{r}$ in the quadratic form $t(u, u ; \lambda)$ by the equation $(\lambda+2) u_{r}+\nabla_{\omega} \cdot u_{\omega}=0$, we obtain the following form in the angular components $u_{\omega}$ :

$$
\begin{aligned}
h\left(u_{\omega}, u_{\omega} ; \lambda\right) & =\left[u_{\omega}, u_{\omega}\right]+\int_{\Omega}\left(\frac{1}{2(1-\lambda)(\lambda+2)}\left|\nabla_{\omega}\left(\nabla_{\omega} \cdot u_{\omega}\right)\right|^{2}\right. \\
& \left.+\left(\frac{1-c \lambda}{\lambda+2}+\frac{\lambda+4}{\lambda+2}\right)\left|\nabla_{\omega} \cdot u_{\omega}\right|^{2}+\frac{1}{2}(\lambda+2)(1-\lambda)\left|u_{\omega}\right|^{2}\right) d \omega .
\end{aligned}
$$

From (8.2) it follows that

$$
m_{j}(\lambda)=\max _{V} \min _{u_{\omega} \in V \backslash\{0\}} \frac{h\left(u_{\omega}, u_{\omega} ; \lambda\right)}{\int_{\Omega}\left(\left|u_{\omega}\right|^{2}+(\lambda+2)^{-2}\left|\nabla_{\omega} \cdot u_{\omega}\right|^{2}\right) d \omega},
$$

where the maximum is taken over all subspaces $V \subset \mathscr{Y}$ of codimension $\geq j-1$.

Lemma 8.1. The equation $m_{j}(\lambda)=0$ has not more than one zero in the interval $[-1 / 2,1)$ for every $j=1,2, \ldots$. If $m_{j}\left(\lambda_{0}\right)=0$ for $\lambda_{0} \in(-1 / 2,1)$, then $m_{j}(\lambda)>0$ for $\lambda \in\left[-1 / 2, \lambda_{0}\right)$ and $m_{j}(\lambda)<0$ for $\lambda \in\left(\lambda_{0}, 1\right)$.

Analogously to [11] we obtain the following methodology to find the eigenvalues of the pencil $\mathfrak{A}(\lambda)$ located in the interval $[-1 / 2,1)$. 
THEOREM 8.1. The number $\lambda_{0} \in[-1 / 2,1)$ is an eigenvalue of the pencil $\mathfrak{U}(\lambda)$ with multiplicity $\nu$ if and only if there exists an integer $k \geq 1$ such that

$$
m_{j}\left(\lambda_{0}\right)=0 \quad \text { for } j=k, k+1, \ldots, k+\nu-1 .
$$

Due to Lemma 8.1 and Theorem 4, the assertions on the eigenvalue $\lambda=1$ can be used to obtain informations on the eigenvalues in the interval $(-1 / 2,1)$.

Consider first the case when $\mathscr{H}_{s}$ is a subspace of the first kind (i.e., $I_{\tau}=\emptyset$.)

TheORem 8.2. Let $\mathscr{H}_{s}$ be a subspace of the first kind. If $k$ is the maximal dimension of subspaces $\mathscr{X} \subset\left\{v \in \mathscr{H}_{s}^{r}: \int_{\Omega} v d \omega=0\right\}$ for which

$$
\int_{\Omega}\left(\left|\nabla_{\omega} v\right|^{2}-6|v|^{2}\right) d \omega<0 \quad \text { for all } v \in \mathscr{X} \backslash\{0\},
$$

then the interval $[-1 / 2,1)$ contains exactly $k$ eigenvalues of $\mathfrak{A}(\lambda)$.

Analogously the following assertion holds in the case when $\mathscr{H}_{s}$ is a subspace of the second kind (i.e. $I_{\tau} \neq \emptyset$.)

THeORem 8.3. Let $\mathscr{H}_{s}$ be a subspace of the second kind. If $k$ is the maximal dimension of the subspace $\mathscr{X} \subset \mathscr{H}_{s}^{r}$ for which

$$
\int_{\Omega}\left(\left|\nabla_{\omega} v\right|^{2}-6|v|^{2}\right) d \omega<0 \quad \text { for all } v \in \mathscr{X} \backslash\{0\},
$$

then the interval $[-1 / 2,1)$ contains exactly $k$ eigenvalues of $\mathfrak{A}(\lambda)$.

As a consequence of Theorem 8.3, the following assertion holds.

COROLlary 8.1. In the case $\mathscr{H}_{s}^{r}=\stackrel{\circ}{H}^{1}(\Omega)$ (this takes place, for example, if only the boundary conditions (i), (iii) appear in the definition of the space $\mathscr{H}$ ), the number of the eigenvalues in $[-1 / 2,1)$ monotonically depends on the domain $\Omega \subset S^{2}$.

\section{Examples.}

1. Let $I_{\tau} \neq \emptyset, I_{n}=\emptyset$. Then $\mathscr{H}_{s}^{r}=\stackrel{\circ}{H}^{1}(\Omega)$, and hence $\mathscr{H}_{s}$ is a subspace of the second kind. Let also $\mathscr{D}_{\alpha}$ be a dihedral angle of aperture $\alpha$.

TheOREM 9.1. If $\mathscr{K} \subset \mathscr{D}_{\alpha}$ with $\alpha \leq \pi / 2$, and $\mathscr{D}_{\alpha} \backslash \overline{\mathscr{K}} \neq \emptyset$, then the strip $-1 / 2 \leq \operatorname{Re} \lambda \leq 1$ does not contain eigenvalues of the pencil $\mathfrak{A}(\lambda)$.

Proof. Let $\Omega_{\alpha}=\mathscr{D}_{\alpha} \cap S^{2}$ and let $\delta$ be the Laplace-Beltrami operator on $S^{2}$. Then the smallest positive eigenvalue of the operator pencil 


$$
-\delta-\lambda(\lambda+1): \stackrel{\circ}{H}^{1}\left(\Omega_{\alpha}\right) \rightarrow H^{-1}\left(\Omega_{\alpha}\right)
$$

is $\pi / \alpha$ (see, e.g., [9]). Hence

$$
\int_{\Omega_{\alpha}}\left|\nabla_{\omega} v\right|^{2} d \omega \geq 6 \int_{\Omega_{\alpha}}|v|^{2} d \omega, \quad v \in \stackrel{\circ}{H}^{1}\left(\Omega_{\alpha}\right),
$$

provided $\alpha \leq \pi / 2$. Since $\Omega \subset \Omega_{\alpha}$ and $\Omega_{\alpha} \backslash \bar{\Omega} \neq \emptyset$, it follows that the inequality (9.1) is valid for $v \in H^{1}(\Omega)$ and the equality in (9.1) may be valid only for $v=0$. Using Theorems 8.3 and 7.2, we obtain the assertion of the theorem.

2. We consider a convex polyhedral cone $\mathscr{K}$ whose boundary $\partial \mathscr{K} \backslash\{0\}$ consists of a finite number of flat open faces $\Gamma_{1}, \ldots, \Gamma_{N}$ and rays. The convexity of $\mathscr{K}$ implies that the angles between two adjacent faces are less than $\pi$. We suppose that the angles between $\Gamma_{1}$ and adjoining faces are less than $\pi / 2$.

Furthermore, we assume that the boundary condition (ii) is prescribed on $\Gamma_{1}$, while the Dirichlet condition is given on the remaining faces.

THEOREM 9.2. Under the above assumptions, the operator pencil $\mathfrak{A}(\lambda)$ has only one eigenvalue $\lambda_{1}=1$ in the strip $-1 / 2 \leq \operatorname{Re} \lambda \leq 1$. This eigenvalue has only the eigenvectors $(0,0,0, c)$, where $c$ is a constant, and there are no generalized eigenvectors.

Proof. We can suppose that the face $\Gamma_{1}$ is located in the plane $x_{3}=0$ and the cone $\mathscr{K}$ is placed in the half-space $x_{3}<0$. Let $\mathscr{K}_{+}$and $\mathscr{K}_{\text {ex }}$ be the following cones:

$$
\mathscr{K}_{+}=\left\{x=\left(x_{1}, x_{2}, x_{3}\right):\left(x_{1}, x_{2},-x_{3}\right) \in \mathscr{K}\right\}, \quad \mathscr{K}_{\text {ex }}=\mathscr{K} \cup \mathscr{K}_{+} \cup \Gamma_{1} .
$$

Clearly, the cone $\mathscr{K}_{e x}$ is also convex. By $\mathfrak{U}_{e x}(\lambda)$ we denote the operator pencil associated with the Dirichlet problem for the operator in $(0.1) \mathscr{K}_{\text {ex }}$.

We consider the auxiliary spectral problem generated by the ratio of quadratic forms:

$$
\frac{\int_{\Omega}\left|\nabla_{\omega} v\right|^{2} d \omega}{\int_{\Omega}|v|^{2} d \omega}, \quad v \in \mathscr{X} \backslash\{0\},
$$

where $\Omega=\mathscr{K} \cap S^{2}$ and

$$
\mathscr{X}=\left\{w \in H^{1}(\Omega): w=0 \text { on } \gamma_{2} \cap \gamma_{3} \cap \cdots \cap \gamma_{N} \text { and } \int_{\Omega} w d \omega=0\right\}
$$


(here $\gamma_{j}=\Gamma_{j} \cap S^{2}$ ). Setting

$$
\hat{v}(\omega)= \begin{cases}v(\omega), & \text { if } \quad \omega_{3}<0, \\ v\left(\omega_{1}, \omega_{2},-\omega_{3}\right), & \text { if } \quad \omega_{3}>0,\end{cases}
$$

we obtain an extension of $v \in \mathscr{X}$ onto $\Omega_{e x}=\mathscr{K}_{e x} \cap S^{2}$. It is evident that

$$
\hat{v} \in\left\{w \in \stackrel{\circ}{H}^{1}\left(\Omega_{e x}\right): \int_{\Omega_{e x}} w d \omega=0\right\} .
$$

Using this extension operator, one can show that the least eigenvalue of the operator induced by (9.2) is not greater than the infimum of the functional (9.2), where $\Omega, v$ are replaced by $\Omega_{e x}, \hat{v}$, respectively, and $\hat{v}$ is defined by (9.3).

Since $\Omega_{e x}$ is placed in a half-sphere, it follows from Proposition 5.1 [11] that the first eigenvalue of the spectral problem in $\Omega_{e x}$ is greater than 6 . Hence the same is valid for the spectral problem in $\Omega$. Now the assertion of the theorem follows from Theorems 8.2 and 7.1 .

\section{REFERENCES}

1. M. Dauge, Stationary Stokes and Navier-Stokes systems on two- and three-dimensional domains with corners. Part I: Linearized equations, SIAM J. Math. Anal. 20 (1989), 74-97.

2. J. Gobert, Une inéquation fondamentale de la théorie de l'élasticité, Bull. Soc. Roy. Sci. Liège No. 3 and 4 (1962).

3. I. I. Gohberg and E. I. Sigal, Operator generalization of the theorem on the logarithmic residue and Rouché's theorem, Matem. Sbornik 84 (1971) 4, 607-629 (English transl. in: Math. USSR Sb. 13 (1971)).

4. L. Hörmander, Linear Partial Differential Operators, Springer-Verlag Berlin-GottingenHeidelberg 1963.

5. V. A. Kondrat'ev, Boundary value problems for elliptic equations in domains with conical or angular points, Trudy Moskov. Mat. Obshch. 16 (1967), 209-292 (English transl. in: Trans. Moscow Math. Soc. 16 (1968)).

6. V. A. Kozlov, On the spectrum of the pencil generated by the Dirichlet problem for elliptic equations in an angle, Siberian Math. J. 32 (1991) 74-87 (Engl. transl. in: Siberian Math. J. 32 (1991) 2, 238-251).

7. V. A. Kozlov, On the Dirichlet problem for elliptic equations on domains with conical points, Diff. Uravn. 26 (1990) 1014-1023 (Engl. transl. in: Diff. Equ. 26 (1990)739-747).

8. V. A. Kozlov and V. G. Maz'ya, Spectral properties of operator pencils generated by elliptic boundary value problems in a cone (in Russian), Functional Analysis and its Applications 22 (1988), 38-46 (Engl. translation in: Functional Anal. Appl. 22 (1988) No. 2, 114 121).

9. V. A. Kozlov and V. G. Maz'ya, On "power-logarithmic" solutions of the Dirichlet problem for elliptic systems in $K_{d} \times \mathrm{R}^{n-d}$, where $K_{d}$ is a d-dimensional cone, Rend. Mat. Acc. Lincei 7 (1996) 9, 17-30.

10. V. A. Kozlov, V. G. Maz'ya and C. Schwab, On singularities of solutions of the displacement problem of linear elasticity near the vertex of a cone, Arch. Rational Mech. Anal. 119 (1992), 197-227. 
11. V. A. Kozlov, V. G. Maz'ya, and C. Schwab, On singularities of solutions to the Dirichlet problem of hydrodynamics near the vertex of a cone J. Reine Angew. Math. 456 (1994), 65-97.

12. V. G. Maz’ya, Sobolev Spaces, Springer-Verlag, Berlin and New York, 1985.

13. V. G. Maz'ya and B. A. Plamenevskij, Estimates in $L_{p}$ and Hölder classes and the MirandaAgmon maximum principle for solutions of elliptic boundary value problems in domains with singular points on the boundary, Math. Nachr. 81 (1978), 25-82 (Engl. translation in: Amer. Math. Soc. Transl 123 (1984)).

14. V. G. Maz'ya and B. A. Plamenevskij, On properties of solutions of three-dimensional problems of elasticity theory and hydrodynamics in domains with isolated singular points, Dinamika Sloshnoi Sredy 50 (1981) 99-120 (Engl. translation in: Amer. Math. Soc. Transl. (2) 123 (1984) 109-123).

15. V. G. Maz'ya, B. A. Plamenevskij and L. I. Stupyalis, The three-dimensional problem of steady-state motion of a fluid with a free surface, Diff. Uravn. i Primenen.-Trudy Sem. Protsessy Optimal. Upravl. 23 (1979) (Engl. transl. in Amer. Math Soc. Transl. 123 (1984) 171-268).

16. R. Temam, Navier-Stokes Equations. North Holland, 1978.

MATEMATISKA INSTITUTIONEN

LINKÖPINGS UNIVERSITET

58183 LINKÖPING

SWEDEN
UNIVERSITÄT ROSTOCK

FACHBEREICH MATHEMATIK

D-18051 ROSTOCK

GERMANY 